\title{
Homaloidal nets and ideals of fat points I
}

\author{
Zaqueu Ramos and Aron Simis
}

\begin{abstract}
We consider plane Cremona maps with proper base points and the base ideal generated by the linear system of forms defining the map. The object of this work is to study the link between the algebraic properties of the base ideal and those of the ideal of these points fattened by the virtual multiplicities arising from the linear system. We reveal conditions which naturally regulate this association, with particular emphasis on the homological side. While most classical numerical inequalities concern the three highest virtual multiplicities, here we emphasize also the role of one single highest multiplicity. In this vein we describe classes of Cremona maps for large and small values of the highest virtual multiplicity. We also deal with the delicate question as to when is the base ideal non-saturated and consider the structure of its saturation.
\end{abstract}

\section{Introduction}

Let $k$ be an infinite field and let $R=k[\mathbf{X}]=k\left[X_{0}, \ldots, X_{n}\right]$ denote a polynomial ring over $k$, endowed with the ordinary standard grading. A rational map $F: \mathbb{P}^{n} \rightarrow \mathbb{P}^{n}$ is defined by a linear system of $n+1$ independent forms of the same degree in $R$. If $F$ is birational then it is called a Cremona map. Beyond the notable modern development on the structure of the Cremona group, a body of results on the nature, structure and uses of an individual Cremona map has lately come up that draws on modern geometric and algebraic tools (for a very short sample, see $[\mathbf{2 - 6}, \mathbf{9}, \mathbf{1 7}-\mathbf{1 9}, \mathbf{2 1}, \mathbf{2 4}, \mathbf{2 5}])$. This paper follows this line and focuses only on the plane case (that is, $n=2$ ).

The core of the work is a study of the intertwining between the algebraic properties of the base locus of $F$, here taken in the sense of the strict base ideal $I \subset R$ generated by the linear system of forms defining $F$, and those of the ideal $I(\mathbf{p}, \boldsymbol{\mu})$ of general fat points $\mathbf{p}$ with the virtual multiplicities $\boldsymbol{\mu}$ of this linear system. A first question concerns how homaloidal virtual multiplicities imply special properties of the corresponding ideal $J \subset R$ of fat points in terms of its Hilbert function and minimal free resolution. Conversely, it is natural to ask how to recover information about the base ideal of the Cremona map out of the features of $J$, such as, for example, the homological structure of the one-dimensional ring $R / I$.

When relating Cremona maps (or, more generally, rational maps of $\mathbb{P}^{2}$ ) to ideals of fat points, a technical difficulty arises at the outset, and that is the fact that the defining linear system of a Cremona map determines infinitely near base points as well as base points in $\mathbb{P}^{2}$. Capturing the entire picture in the passage to the current notion of fat points becomes flawed. To partially overcome this problem, it has been proposed in [14] to introduce a new ideal: that is, given a Cremona map of degree $d \geqslant 1$, with cluster $\mathcal{K}$ of points and virtual multiplicities, one lets $\ell_{\mathcal{K}}(d) \subset R_{d}$ consist of all forms $f$ of degree $d$ such that the curve $C=V(f)$ passes virtually through $\mathcal{K}$, and sets $I_{\mathcal{K}}:=\bigoplus_{d \geqslant 0} \ell_{\mathcal{K}}(d) \subset R$, which is a nice homogeneous ideal. As it

Received 18 June 2015

2010 Mathematics Subject Classification 13D02, 13H10, 13H15, 14E05, 14E07, 14M05 (primary), 13A02, 13C14, 14C20 (secondary).

The first author was partially supported by a CNPq post-doc fellowship (151229/2014-7) and the second author was partially supported by a CNPq grant (302298/2014-2) and by a CAPES-PVNS Fellowship. 
turns out, there are inclusions $I \subset I_{\mathcal{K}} \subset I(\mathbf{p}, \boldsymbol{\mu})$, where $\mathbf{p}$ are the proper points of the map and $\boldsymbol{\mu}$ are the corresponding virtual multiplicities. Moreover, the rightmost inclusion is an equality if the map has only proper points (that is, a simple Cremona map in the classical terminology [1]). Of course, the hardship in capturing this construction in purely algebraic terms is the notion of 'passing virtually through $\mathcal{K}$ ' (for the details see [14, Introduction]).

A similar notion has been given by Harbourne in [13] in terms of the push-down of a suitably defined divisor on the blowup of points on $\mathbb{P}^{2}$. If there is an explicit ideal of $R$ to be extracted from the latter notion it ought to be very similar, if not the same, as the one in [14].

Dealing with these additional data would certainly cloud the central idea of this paper. Therefore, we assume that base points are points of $\mathbb{P}^{2}$ and, as a rule, general points. Since Cremona maps densely exist in this context of points, for any proper homaloidal type, we will be dealing with a vast universe. In other words, we will emphasize the role of the proper homaloidal type and of a Cremona map with this type, based on proper points only. Of course, any set of points with random coordinates is considered to be general. In the present context, the choice of sufficiently general points could be more precisely described in terms of avoiding the zero set of minors of a certain matrix (see, for example, [15]). For ordinary computer calculation, by and large random point coordinates are taken, though special scripts are available for the second alternative (see, for example, $[\mathbf{1 2}]$ ).

For plane Cremona maps of degree at least two, asking when the base ideal $I \subset R$ is saturated is tantamount to asking when $R / I$ is a Cohen-Macaulay ring: that is, when $I$ is generated by the maximal minors of a $3 \times 2$ homogeneous matrix with entries in $R$. We will often imprecisely refer to a saturated or Cohen-Macaulay Cremona map in such a situation. While there are many rational maps on $\mathbb{P}^{2}$, in any degree, defined by such ideals, the question for Cremona maps becomes tighter.

As is usually taken for granted while discussing this sort of questions, we assume throughout that the base field $k$ is algebraically closed (a few times of characteristic zero).

We now proceed to a more detailed description of the sections.

Section 1 is a brief account of the preliminaries and the terminology; the subsection titles speak for themselves. We have tried to be as self-contained as possible in terms of the usage of these two aspects.

Section 2 contains the main theorem that connects the base ideal $I \subset R$ of a plane Cremona map of degree $d \geqslant 4$ with proper points and the associated ideal $J \subset R$ of fat points. The minimal free $R$-resolution of $J$ is made explicit and it is shown that the linear system $J_{d+1}$ defines a birational map onto its image. The proofs of both results are entirely ideal theoretic, but we have added an explanation, through the calculation of the regularity and a typical property of Cremona maps, as to how the second of these results is included in a theorem of Geramita et al. [10].

We also present a substantial list of homological results relating the two ideals $I \subset J$. For example, it is explained how to get to the free resolution of $I$ from the free resolution of $J$. In addition, it is shown that if the $R$-module $J / I$ has dimension one, then the CohenMacaulayness of $R / I$ is tantamount to that of $J / I$, which is a simplification of the theory since the latter is a linearly presented $R$-module. On the other hand, it is shown that the condition $\operatorname{dim}(J / I)=0$ is equivalent to $J$ being the saturation of $I$, which, in turn, in degree greater than or equal to four, can only happen for the homaloidal types $\left(4 ; 2^{3}, 1^{3}\right)$ and $\left(5 ; 2^{6}\right)$.

In $\S 3$ we start a classification of homaloidal types according to the highest virtual multiplicity $\mu=\mu_{1}$. The permeating idea is the expectation that, for $\mu_{1} \geqslant d-4$, any Cremona map on general points of such a homaloidal type is saturated. While this is sufficiently known for $\mu=d-1$ (de Jonquières case) we prove this for $\mu=d-2$ using the apparatus of the previous section. This is obtained by showing that the linear matrix $\mathcal{L}$ presenting the ideal $J / I$ has Fitting $I_{1}(\mathcal{L})$ of minimal codimension. Alas, we also show, to our regret, that this condition fails for $\mu \leqslant d-3$. 
There is, however, a set of evidence pointing to saturation in the cases where $d-4 \leqslant \mu \leqslant d-3$. In fact, we are naturally led to (loosely) conjecture that the Cremona map on general (proper) points is saturated unless $\mu \leqslant\lfloor d / 2\rfloor$.

The last section is a detailed study of a strong positive case of the above conjecture, namely, the case of a homaloidal type $T$ whose virtual multiplicities are all even. This consideration unexpectedly stretches to a close look at the type (1/2) $T$ obtained from $T$ by dividing all multiplicities by two. We convey that, although $(1 / 2) T$ is not a homaloidal type, it, nevertheless, satisfies similar 'equations of condition' (the terminology sub-homaloidal has been introduced to designate a set of virtual multiplicities satisfying these new numerical conditions).

We note that passing to the corresponding ideals of fat points for $T$ and $(1 / 2) T$, the first of these ideals is the second symbolic power of the second ideal. This in itself allows for a crosscut between properties of the two and sets up a relation between the respective initial degrees. A finer analysis will be developed in a sequel to this paper, where the order of priority is subverted, enhancing properties of the second of these ideals.

This part culminates with a proof that the base ideal $I$ of a Cremona map $\mathfrak{F}: \mathbb{P}^{2} \rightarrow \mathbb{P}^{2}$ of degree $d$, whose virtual multiplicities are all even, is not saturated under the hypothesis that there are three highest virtual multiplicities equal to $\lfloor d / 2\rfloor$. In addition, we give a minimal set of explicit generators concocted from the respective equations of three irreducible fundamental curves relative to base points of $\mathfrak{F}^{-1}$. Finally, we prove that the saturation of $I$ requires only one additional generator and that this generator can be made as explicit as possible within the context.

As a pointer, the following are the main results of the paper: Theorem 2.6, Propositions 2.8, 3.1, 3.2, 3.4, 4.6, Theorem 4.11 and Proposition 4.12 .

\section{Recap of terminology}

In this section we briefly summarize the basics of linear systems and of ideals of fat points that are going to be used in the subsequent work. For complete details, see [1] or [14, Introduction].

\subsection{Linear systems, base points and Cremona maps}

By a linear system of plane curves of degree $d$ we mean a $k$-vector subspace $L_{d}$ of the vector space of forms of degree $d$ in the standard graded polynomial ring $R:=k[x, y, z]$. A linear system $L_{d}$ defines a rational map $\mathfrak{L}_{d}$ with source $\mathbb{P}^{2}$ and target $\mathbb{P}^{r}$, where $r+1$ is the vector space dimension of $L_{d}$. As such, both $L_{d}$ and its closely associated $\mathfrak{L}_{d}$ have a fixed part and a set of base points. More algebraically, we set $I:=\left(L_{d}\right) \subset R$, the ideal generated by the elements of $L_{d}$. Write $I=(I: F) F$, where $F \in R$ is, up to non-zero scalars, a uniquely defined form of degree $\leqslant d$ such that $I: F$ has codimension greater than or equal to two. In other words, $F$ is the gcd of a set of generators of $I$ and $I: F=(1 / F) I$. Then $F$ (or its zero set in $\left.\mathbb{P}^{2}\right)$ is the fixed part of the system, while $V(I: F) \subset \mathbb{P}^{2}$ is a finite set since $I: F$ is an ideal of codimension $\geqslant 2$ (the elements of $V(I: F)$ are the proper base points of $L_{d}$ or of the corresponding rational map). We say that the linear system has no fixed part or is without fixed part, meaning that $\operatorname{deg}(F)=0$ or, equivalently, that $I$ has codimension greater than or equal to two.

Throughout this work, $I: F$ has codimension exactly two as, otherwise, the set of base points would be empty. Then $I: F$ has a primary decomposition whose components are primary ideals associated to the minimal primes $\left\{P_{1}, \ldots, P_{n}\right\}$ of $R / I: F$ defining the base points $V(I: F)=\left\{p_{1}, \ldots, p_{n}\right\}$. Since $k$ is algebraically closed, every one of these primes is generated by two independent linear forms. We remark that, in the tradition of rational maps, the fixed part is often set aside because the generators of $I$ essentially define the same map 
as the generators of $I: F$. However, in the theory of linear systems, it is often the case that linear systems without fixed part have subsystems with non-trivial fixed part.

For the sake of self-containment, we recall the notion of multiplicity or local vanishing order. Namely, given a variety $X$, a smooth point $p \in X$ and a hypersurface (divisor) $D$ then the (effective) multiplicity $e_{p}(D)$ of $D$ at $p$ is the order of vanishing of a local equation of $D$ at $p$ : algebraically, if $f$ is a local equation of $D$ at $p$, then

$$
e_{p}(D)=\max \left\{s \geqslant 0 \mid f \in \mathfrak{m}_{p}^{s}\right\}
$$

where $\mathfrak{m}_{p}$ is the maximal ideal of the local ring of $X$ at $p$. For our purpose, $X$ will always be a projectively embedded surface and, in fact, mostly $\mathbb{P}^{2}$ or the blowup of a set of points therein.

The virtual multiplicity of $L_{d}$ at one of its proper base points $p$ is the non-negative integer

$$
\mu_{p}=\mu_{p}\left(L_{d}\right):=\min \left\{e_{p}(f) \mid f \in L_{d}\right\}=\min _{f \in L_{d}}\left\{s_{f} \geqslant 0 \mid f \in\left(P_{P}\right)^{s_{f}} \backslash\left(P_{P}\right)^{s_{f}+1}\right\} .
$$

(Note that requiring the global containment $f \in P^{s_{f}} \backslash P^{s_{f}+1}$ in the definition is equivalent, since each $P$ is a complete intersection.) Clearly, the effective multiplicity of an element of $L_{d}$ at a proper base point $p$ is at least $\mu_{p}$, while the subset of $L_{d}$ whose elements have effective multiplicity equal to $\mu_{p_{j}}$ forms an open set $U_{p_{j}}$ (in the set of coefficients as parameters). Also, $L_{d}$ admits infinitely near base points, in contrast to its proper base points, and the virtual multiplicity at such points can be defined in a similar way by blowing up proper points.

An important property of a plane Cremona map is that its virtual multiplicities satisfy the classical equations of condition (see $[\mathbf{1}, 2.5]$ ): that is,

$$
\sum_{p} \mu_{p}=3 d-3, \quad \sum_{p} \mu_{p}^{2}=d^{2}-1
$$

where $p$ runs through the set of (proper and infinitely near) base points of the corresponding $L_{d}$ with respective multiplicities $\mu_{p}$. An abstract configuration $\left(d ; \mu_{1}, \ldots, \mu_{r}\right)$ satisfying the equations of condition is called a homaloidal type. A homaloidal type is called proper if there exists a plane Cremona map with this type. There is an important practical tool to test whether a given homaloidal type is proper - it is called the Hudson test [1, Corollary 5.3.2]. This idea has been largely developed by Nagata in [16]. Geometrically, the test can simply be translated into composing the rational map with the standard quadratic map based on three points of highest virtual multiplicities. Yet the encoded arithmetic and algebra keep a few surprises.

\subsection{Ideals of fat points}

Besides the interest in the role of linear systems in the study of rational maps, there is a vast literature on the largest possible (complete) such systems for a fixed degree: the so-called ideals of fat points.

For convenience, recall the definition. We are given a set $\mathbf{p}=\left\{p_{1}, \ldots, p_{n}\right\} \subset \mathbb{P}^{2}$ of points and respective appended multiplicities $\boldsymbol{\mu}=\left\{\mu_{1}, \ldots, \mu_{n}\right\}, \mu_{j} \in \mathbb{N}_{+}$. Let $L_{d}(\mathbf{p} ; \boldsymbol{\mu}$ ) (for a lack of shorter notation) denote the set of all $d$-forms $f \in R$ such that $e_{p_{j}}(f) \geqslant \mu_{j}$ for every $j$. Because $e_{p_{j}}(f)$ has the properties of a valuation, it follows, immediately, that this set is a vector space, that is, it is a linear system of plane curves of degree $d$. By definition, it is the largest possible such linear system with $\mathbf{p}$ as its set of proper base points and $\mu_{j}, 1 \leqslant j \leqslant n$ as the corresponding virtual multiplicities and, actually, it contains as a vector space all such vector subspaces. If we let $d$ run through $\mathbb{N}$, we obtain the $\boldsymbol{\mu}$-fat ideal of $\mathbf{p}$, given by

$$
I(\mathbf{p} ; \boldsymbol{\mu}):=\bigoplus_{d \in \mathbb{N}} L_{d}(\mathbf{p} ; \boldsymbol{\mu}) \simeq \bigcap_{j=1}^{n} P_{j}^{\mu_{j}},
$$

where $P_{j} \subset R$ is the homogeneous prime ideal of the point $p_{j}$. 
Clearly, the $\boldsymbol{\mu}$-fat ideal has codimension two. However, the ideal $\left(L_{d}(\mathbf{p} ; \boldsymbol{\mu})\right) \subset R$ generated in a single degree $d$ is often of codimension one. If the points $\mathbf{p}$ are general, then some authors write $I(n ; \boldsymbol{\mu})$ instead, a notation we avoid due to the diversity of notions of general position. We will say general points (or even sufficiently general points) in the sense that they avoid a proper closed subset of $\mathbb{P}^{2} \times \ldots \times \mathbb{P}^{2}$ ( $n$ factors $)$. This vague notion can be made more precise (see, for example, $[\mathbf{1 5}]$ ). In any case, for the explicit computation, one can always take a random set of points.

The ideal $I(\mathbf{p} ; \boldsymbol{\mu})$ is supple in some senses. Firstly, it is a perfect ideal: that is, the ring $R / I(\mathbf{p} ; \boldsymbol{\mu})$ is Cohen-Macaulay. Secondly, the degree of the corresponding scheme is automatic and depends only on the number of points and their appended multiplicities: that is,

$$
e(R / I(\mathbf{p} ; \boldsymbol{\mu}))=\sum_{j=1}^{n} \frac{\mu_{j}\left(\mu_{j}+1\right)}{2} .
$$

Thirdly, it is an integrally closed ideal. Further, quite a bit is known about the Hilbert function of $R / I(\mathbf{p} ; \boldsymbol{\mu})$ and its graded Betti numbers. More generally, we have the following.

Let $J \subsetneq R:=k[x, y, z]$ denote an unmixed homogeneous ideal of codimension two. Since $R / J$ is Cohen-Macaulay the minimal graded $R$-resolution of $J$ has the form

$$
0 \rightarrow F_{1} \stackrel{\varphi}{\longrightarrow} F_{0} \longrightarrow J \rightarrow 0,
$$

where $\varphi$ is a homogeneous map and $F_{0}, F_{1}$ are free graded modules.

Let $h_{R / J}(t):=\operatorname{dim}_{k}\left(R_{t} / J_{t}\right)(t \geqslant 0)$ stand for the Hilbert function of $R / J$. Recall that the Hilbert polynomial of $R / J$ is of degree zero and coincides with the multiplicity $e(R / J)$.

The following facts are well known.

(a) $h_{R / J}(t)$ is strictly increasing until it reaches its maximum $e(R / J)$ and thereon stabilizes.

(b) The least $t$ such that $h_{R / J}(t)=e(R / J)$ is the regularity index of $R / J$.

(c) Moreover, set $F_{0}=\bigoplus_{t>0} R(-t)^{n_{t}}$ so that the minimal number of generators of $J$ is $\sum_{t>0} n_{t}$. Then $n_{t}=\operatorname{dim}_{k} \operatorname{coker}\left(R_{1} \otimes_{k} J_{t-1} \longrightarrow J_{t}\right)=\operatorname{dim}_{k}\left(J_{t}\right)-\operatorname{dim}_{k}\left(R_{1} J_{t-1}\right)$, where $R_{1} J_{t-1}$ is short for the vector $k$-subspace of $J_{t}$ spanned by the products of $x, y, z$ by the elements of a basis of $J_{t-1}$, and $R_{1} \otimes_{k} J_{t-1} \longrightarrow J_{t}$ denotes the natural multiplication map.

\section{Ideals of fat points coming from homaloidal types}

Recall that a net of degree $d$ is a linear system $L_{d}$ spanned by three independent forms in $R_{d}=k[x, y, z]_{d}$ without a proper common factor. We say that the net defined by $L_{d}$ is complete if $L_{d}=J_{d}$, where $J=I(\mathbf{p} ; \boldsymbol{\mu})$ is the ideal of fat points based on the cluster $(\mathbf{p} ; \boldsymbol{\mu})$ of proper points defined by $L_{d}$. Note that completeness has to do with a fixed degree $d$, for which it implies equalities $\left(L_{d}\right)=\left(I_{\mathcal{K}}\right)_{d}=I(\mathbf{p}, \boldsymbol{\mu})_{d}$, in the notation explained in the Introduction. We say that the net $L_{d}$ is homaloidal if it defines a Cremona map of $\mathbb{P}^{2}$.

The moral of this section is supported by the following basic result.

Proposition $2.1(\operatorname{char}(k)=0)$. Let $L_{d}$ denote a homaloidal net and $T:=\left(d ; \mu_{1}, \ldots, \mu_{r}\right)$ be the corresponding proper homaloidal type. Then:

(a) $L_{d}$ is complete; and

(b) there exists a non-empty Zariski open subset $U \subset \underbrace{\mathbb{P}^{2} \times \ldots \times \mathbb{P}^{2}}_{r}$ such that the coordinates of any tuple of points in $U$ is the set of the base points of a simple Cremona map with homaloidal type $T$ (that is, informally speaking, that choosing any set of $r$ 'general' points in $\mathbb{P}^{2}$ will give rise to a complete net as in (a)). 
Proof. (a) See [1, Proposition 2.5.2]. A more conceptual proof follows by the Enriques criterion [1, Proposition 5.1.1], as follows. Let $L_{d}$ span a homaloidal net of degree $d$ defining the given Cremona map. Set $J:=I(\mathbf{p} ; \boldsymbol{\mu})$. Clearly, $L_{d} \subset J_{d}$. Since $L_{d}$ is the linear system of a Cremona map, its generic member $f$ is irreducible. But $f \in J_{d}$, and hence the Enriques criterion implies that $J_{d}$ is a homaloidal system, that is, the linear system of a Cremona map. In particular, $\operatorname{dim}_{k}\left(J_{d}\right)=3$, so the proof is complete.

(b) See [1, Theorem 5.2.19]).

REMARK 2.2. One cannot possibly be fooled by the apparent amplitude of the Enriques criterion: the existence of irreducible curves in the linear system is an expression of Bertini's theorem, even without the assumption that the type $T=(d ; \boldsymbol{\mu})$ be homaloidal (see $[\mathbf{1 1}$, Proposition 9]) for cases in which, in fact, every member is irreducible.

\subsection{Main theorem}

In this part we discuss the homological nature of the ideal of fat points of homaloidal type.

We start with a basic result.

Lemma 2.3. Let $\mathfrak{F}: \mathbb{P}^{2} \rightarrow \mathbb{P}^{2}$ be a Cremona map with homaloidal type $(d ; \boldsymbol{\mu})$ whose base points $\mathbf{p}$ are proper (that is, $\left.\mathbf{p} \subset \mathbb{P}^{2}\right)$ and let $J:=I(\mathbf{p}, \boldsymbol{\mu}) \subset R:=k[x, y, z]$ denote the associated ideal of fat points. Then:

(i) the initial degree of $J$ is $d$;

(ii) the Hilbert function of $R / J$ is maximal with $h_{R / J}(t)=e(R / J)$ for $t \geqslant d$ (that is, the regularity index of $R / J$ is $d$ ); and

(iii) $n_{t}=0$ for $t \geqslant d+2$.

Proof. (i) For this assertion, suppose that $0 \neq f \in J_{d-1}$. Then $\{x f, y f, z f\}$ is a $k$-linearly independent subset of $J_{d}$. Since $\mathfrak{F}$ is a Cremona map, its underlying two-dimensional linear system $L_{d}$ is complete, by Proposition 2.1: that is, $L_{d}=J_{d}$. Therefore, $\{x f, y f, z f\}$ is a $k$-basis of $L_{d}$, which contradicts the fact that $L_{d}$ has no fixed part.

(ii) For the second assertion, we recall that the equations of the conditions satisfied by a Cremona map and the knowledge that $\operatorname{dim}_{k}\left(J_{d}\right)=\operatorname{dim}_{k}\left(L_{d}\right)=3$ imply that

$$
\operatorname{dim}_{k}\left(J_{d}\right)=\frac{(d+2)(d+1)}{2}-\sum \frac{\left(\mu_{i}+1\right) \mu_{i}}{2} .
$$

Since $d$ is the initial degree of $J$, the Hilbert function of $R / J$ is maximal.

(iii) This follows from (ii) (see [7]).

We will need the following structural result which could not be found in this exact form in the literature.

Proposition 2.4. Let $\mathfrak{F}: \mathbb{P}^{2} \rightarrow \mathbb{P}^{2}$ be a Cremona map whose base ideal is perfect and has a linear syzygy. Then $\mathfrak{F}$ is a de Jonquières map.

Proof. Since any quadratic Cremona map is de Jonquières, we may assume, at the outset, that $\mathfrak{F}$ has degree greater than or equal to three. Consider the presentation matrix of its base ideal $I$, given by

$$
L:=\left(\begin{array}{cc}
\ell_{1} & q_{1} \\
\ell_{2} & q_{2} \\
\ell_{3} & q
\end{array}\right),
$$

where $\ell_{i}$ is a linear form and $q_{i}, q$ are forms of degree greater than or equal to two. By [8, Proposition 3.4], $I$ is not an ideal of linear type. It follows that the codimension of the 
ideal generated by the entries of $L$ is less than or equal to two since, otherwise, $I$ would be generically a complete intersection; but an almost complete intersection which is generically a complete intersection must be of linear type [23, Proposition 3.7]. In particular, the three linear forms along the first column cannot be $k$-linearly independent. By a suitable elementary operation, we may assume that $\ell_{1}=x, \ell_{2}=y, \ell_{3}=0$. Then one has $I=\left(x q, y q, \ell_{1} q_{2}-\ell_{2} q_{1}\right)$. Moreover, by [18, Corollaire 2.3], $q$ and $\ell_{1} q_{2}-\ell_{2} q_{1}$ are relatively prime $z$-monoids. This means that $\mathfrak{F}$ is a de Jonquières map (see $[\mathbf{2 0}$, Introduction]).

REMARK 2.5. There are examples of non-Cremona dominant rational maps $\mathbb{P}^{2} \rightarrow \mathbb{P}^{2}$ with perfect base ideal $I$ and unique linear syzygy (up to scalars). Such examples are written down where, in addition, $I$ is an ideal of linear type (equivalently, such that the three coordinates of the linear syzygy are $k$-linearly independent).

We are now ready for the main result of this part.

Theorem 2.6. Let $\mathfrak{F}: \mathbb{P}^{2} \rightarrow \mathbb{P}^{2}$ be a Cremona map with homaloidal type $(d ; \boldsymbol{\mu})$, with $d \geqslant 4$, whose base points $\mathbf{p}$ are proper (that is, $\mathbf{p} \subset \mathbb{P}^{2}$ ) and let $I \subset R$ denote its base ideal. Let $J:=I(\mathbf{p}, \boldsymbol{\mu})$ denote the associated ideal of fat points.

(a) The minimal graded free resolution of $J$ is of the form

$$
0 \rightarrow R(-(d+2))^{d-2} \oplus R(-(d+1))^{s} \stackrel{\varphi}{\rightarrow} R(-(d+1))^{d-4+s} \oplus R(-d)^{3} \rightarrow J \rightarrow 0,
$$

where $s$ is the number of independent linear syzygies of the ideal I. Furthermore, $0 \leqslant s \leqslant 1$, while $s=1$ if and only if $\mathfrak{F}$ is a de Jonquières map.

(b) The linear system $J_{d+1}$ defines a birational mapping of $\mathbb{P}^{2}$ onto the image in $\mathbb{P}^{d+4+s}$.

Proof. (a) By Lemma 2.3(iii), we know that $J$ has only minimal generators in degrees $d$ and $d+1$ and that, in degree $d$, the minimal number of generators is three. Thus, we only have to determine the minimal number of generators $n_{d+1}$.

By Lemma 2.3(ii) and by the equations of condition,

$$
\operatorname{dim}_{k}\left(J_{d+1}\right)=\frac{(d+3)(d+2)}{2}-\sum \frac{\left(\mu_{i}+1\right) \mu_{i}}{2}=d+5 .
$$

Let $N:=\operatorname{ker}\left(R_{1} \otimes_{k} J_{d} \longrightarrow J_{d+1}\right)$ and $K:=\operatorname{coker}\left(R_{1} \otimes_{k} J_{d} \longrightarrow J_{d+1}\right)$. From the exact sequence

$$
0 \rightarrow N \longrightarrow R_{1} \otimes_{k} J_{d} \longrightarrow J_{d+1} \longrightarrow K \rightarrow 0
$$

and, since $\operatorname{dim}_{k}\left(R_{1} \otimes_{k} J_{d}\right)=9$, we then get $n_{d+1}=d-4+\operatorname{dim}_{k}(N)$.

We claim that $\operatorname{dim}_{k}(N)$ coincides with the number of independent linear syzygies of the ideal $I$. For this to be seen, let $\left\{D_{1}, D_{2}, D_{3}\right\}$ denote a $k$-vector basis of $J_{d}$. Letting $\left\{T_{1}, T_{2}, T_{3}\right\}$ denote indeterminates over $R$, one has $k\left[T_{1}, T_{2}, T_{3}\right]_{1}=k T_{1}+k T_{2}+k T_{3} \simeq J_{d}$ as $k$-vector spaces. Therefore, $R_{1} \otimes_{k} J_{d} \simeq R_{1} \otimes_{k} k\left[T_{1}, T_{2}, T_{3}\right]_{1} \simeq R\left[T_{1}, T_{2}, T_{3}\right]_{(1,1)}$ as $k$-vector spaces, where the latter is the graded part in bidegree $(1,1)$ of the standard bigraded polynomial ring $R\left[T_{1}, T_{2}, T_{3}\right]$. With this identification, it follows, immediately, that $N$ coincides with the bigraded (1,1)-part of the ideal generated by the bigraded relations of $\left\{D_{1}, D_{2}, D_{3}\right\}$ (the socalled Rees ideal of $\left.I=\left(D_{1}, D_{2}, D_{3}\right)\right)$ which is identified with the $k$-vector space spanned by the $R$-linear syzygies of $I$.

This shows the first statement in (a).

For the supplementary statement, let $s$ denote (as in the statement) the number of independent linear syzygies of $I$. Clearly, this is exactly the number of independent $k$-linear relations among the elements $\left\{x D_{j}, y D_{j}, z D_{j}\right\}$ spanning $R_{1} J_{d}$. Thus, to show that $0 \leqslant s \leqslant 1$, 
it suffices to prove that these forms span a $k$-vector space of dimension at least eight. For this purpose, consider a linear form $\ell \in R$ which is a non-zero divisor on $R / J$. We claim that $x D_{1}, x D_{2}, x D_{3}, \ell D_{1}, \ell D_{2}, \ell D_{3}$ are $k$-linearly independent. Indeed, from the relation

$$
\alpha_{1} x D_{1}+\alpha_{2} x D_{2}+\alpha_{3} x D_{3}+\beta_{1} \ell D_{1}+\beta_{2} \ell D_{2}+\beta_{3} \ell D_{3}=0,
$$

with $\alpha_{j}, \beta_{j} \in k$, one deduces that

$$
x\left(\alpha_{1} D_{1}+\alpha_{2} D_{2}+\alpha_{3} D_{3}\right)=\ell\left(-\beta_{1} D_{1}-\beta_{2} D_{2}-\beta_{3} D_{3}\right) ;
$$

and hence

$$
\left(-\beta_{1} D_{1}-\beta_{2} D_{2}-\beta_{3} D_{3}\right) / x=f \in k[x, y, z],
$$

with $f$ a form of degree $d-1$. Noting that $\ell f \in\left(D_{1}, D_{2}, D_{3}\right) \subset J$, since $\ell$ is regular modulo $J$ we must have $f \in J$. But as the initial degree of $J$ is $d$, we conclude that $f=0$. Substituting this into the above relations gives

$$
\alpha_{1} D_{1}+\alpha_{2} D_{2}+\alpha_{3} D_{3}=0 \quad \text { and } \quad \beta_{1} D_{1}+\beta_{2} D_{2}+\beta_{3} D_{3}=0
$$

so forcing $\alpha_{1}=\alpha_{2}=\alpha_{3}=\beta_{1}=\beta_{2}=\beta_{3}=0$ as $\left\{D_{1}, D_{2}, D_{3}\right\}$ is $k$-linearly independent.

We have proved that $\operatorname{dim}_{k}\left(R_{1} J_{d}\right) \geqslant 6$. We can easily do better, that is, force $\ell$ to avoid the prime $(x, y)$ as well. We claim that, for some $1 \leqslant j \leqslant 3$, one has $y D_{j} \notin k x D_{1}+k x D_{2}+$ $k x D_{3}+k \ell D_{1}+k \ell D_{2}+k \ell D_{3}$. Suppose the contrary, in particular, $y D_{j} \in(x, \ell)$ for $1 \leqslant j \leqslant 3$. But, since $\ell \notin(x, y)$ by our choice, $y \notin(x, \ell)$ and hence $D_{j} \in(x, \ell)$ for every $j \in\{1,2,3\}$. It follows that $(x, \ell)$ is a minimal prime of $k[x, y, z] /\left(D_{1}, D_{2}, D_{3}\right)$, and thus a minimal prime of $R / J$ as well, which contradicts the choice of $\ell$.

This gives $\operatorname{dim}_{k}\left(R_{1} J_{d}\right) \geqslant 7$.

We still have one to go. Thus, assume $\operatorname{dim}_{k}\left(R_{1} J_{d}\right)=7$, that is, the ideal $I$ has two independent linear syzygies. Then the syzygy matrix of $J$ would have the form

$$
\varphi=\left(\begin{array}{cc|cccc}
\ell_{1,1} & \ell_{1,2} & q_{1,1} & q_{1,2} & \ldots & q_{1, d-2} \\
\ell_{2,1} & \ell_{2,2} & q_{2,1} & q_{2,2} & \ldots & q_{2, d-2} \\
\ell_{3,1} & \ell_{3,2} & q_{3,1} & q_{3,2} & \ldots & q_{3, d-2} \\
\hline 0 & 0 & h_{1,1} & h_{1,2} & \ldots & h_{1, d-2} \\
0 & 0 & h_{2,1} & h_{2,2} & \ldots & h_{2, d-2} \\
0 & 0 & h_{3,1} & h_{3,2} & \ldots & h_{3, d-2} \\
\vdots & \vdots & \vdots & \vdots & & \vdots \\
0 & 0 & h_{d-2,1} & h_{d-2,2} & \ldots & h_{d-2, d-2}
\end{array}\right) .
$$

Now, note that for each $1 \leqslant j \leqslant 3$, the generator $D_{j}$ can be taken to be the determinant of the submatrix of $\varphi$ obtained by omitting the $j$ th row. This gives that the determinant of the right lower corner of size $(d-2) \times(d-2)$ is a factor of every $D_{j}$. But this is absurd as we are assuming $d \geqslant 5$ and the linear system $J_{d}$ has no fixed part. Therefore, $\operatorname{dim}_{k}\left(R_{1} J_{d}\right) \geqslant 8$, as intended.

To complete the proof of the supplementary assertions of this item we explain the issue about the de Jonquières map. From one side the structure of a plane de Jonquières map [14, Proposition 2.3] tells us that its base ideal admits a unique linear syzygy up to scalars, that is, $s=1$ in the notation of (a).

Conversely, assume that $s=1$. We now focus our attention on the $(d-3) \times(d-1)$ submatrix $\mathfrak{L}$ of the matrix $\varphi$ obtained by omitting the first three rows. 
We note that $\mathfrak{L}$ has the form $\left(\mathbf{0} \mid \mathfrak{L}^{\prime}\right)$, where $\mathbf{0}$ is the $(d-3) \times 1$ zero matrix and $\mathfrak{L}^{\prime}$ is a $(d-3) \times(d-2)$ matrix with linear entries. The ideal of maximal minors of the latter matrix has codimension greater than or equal to two as it contains $I$. It follows readily that the complex

$$
0 \rightarrow R \longrightarrow R^{d-2} \stackrel{\mathfrak{L}^{\prime}}{\longrightarrow} R^{d-3}
$$

is exact, where the entries of the leftmost map are the (signed, ordered) maximal minors of $\mathfrak{L}^{\prime}$.

On the other hand, the syzygies $\mathfrak{K}$ of $\mathfrak{L}=\left(\mathbf{0} \mid \mathfrak{L}^{\prime}\right)$ are $\left(\begin{array}{llll}1 & \ldots \ldots 0)^{t}\end{array}\right.$ and the syzygies of $\mathfrak{L}^{\prime}$. It follows that $\mathfrak{K}$ is a matrix with two columns. Therefore, by Proposition 2.8(iv) below, the syzygies of the ideal $I$ are generated by two syzygies, that is, $I$ is a perfect ideal.

Now apply Proposition 2.4.

(b) The proof will consist of showing that $\left(J_{d+1}\right)$ has maximal analytic spread (equal to three) and is linearly presented. Then the stated result follows from [8, Theorem 3.2].

Since $\left(J_{d+1}\right)$ is equi-homogeneous, its analytic spread is the dimension of the $k$-algebra $k\left[J_{d+1}\right]$. But $k\left[x D_{1}, y D_{1}, z D_{1}\right]$ is a $k$-subalgebra of $k\left[J_{d+1}\right]$, while $\left\{x D_{1}, y D_{1}, z D_{1}\right\}$ is obviously algebraically independent over $k$. Therefore, $\operatorname{dim} k\left[J_{d+1}\right]=3$.

To proceed, we assume, for simplicity, that the Cremona map is not a de Jonquières map; the result is similar in the latter case and the changes are tiny. We actually show that $\left(J_{d+1}\right)$ has a free linear resolution of the shape

$$
0 \rightarrow R(-(d+3))^{3} \longrightarrow R(-(d+2))^{d+7} \longrightarrow R(-(d+1))^{d+5} \longrightarrow\left(J_{d+1}\right) \rightarrow 0 .
$$

To see this, we argue as follows. Note that $J=\left(J_{d}, B\right)$ and $\left(J_{d+1}\right)=\left(R_{1} J_{d}, B\right)$, where $B$ is a $k$-vector basis of the direct supplement of $\operatorname{Im}\left(R_{1} \otimes_{k} J_{d} \longrightarrow J_{d+1}\right)$. By (a), it follows that $\left(J_{d+1}\right)$ is generated by $9+d-4=d+5$ forms of degree $d+1$. Furthermore, again by (a), any minimal syzygy of $J$ is the transpose of a vector $\left(q_{1} q_{2} q_{3} \ell_{4} \ldots \ell_{d-1}\right)$, where each $q_{i}$ is a two-form and each $\ell_{j}$ is a linear form.

Writing every $q_{i}$ as an element of $\mathfrak{m}:=(x, y, z)$ with linear coefficients readily yields a linear syzygy of the above generators of $\left(J_{d+1}\right)$ (the expression of $q_{i}$ as an element of $\mathfrak{m}$ is not unique, but it will turn out to be irrelevant which one is picked). This gives a total of $d-2$ linear syzygies.

One has to add up the reduced Koszul relations of each one of the following pairs $\left\{x D_{i}, y D_{i}\right\},\left\{x D_{i}, z D_{i}\right\},\left\{y D_{i}, z D_{i}\right\}$, for $i=1,2,3$. These contribute nine additional syzygies, giving a total of $d-2+9=d+7$ syzygies. Note that the latter syzygies are just the diagonal sum of three times the matrix of Koszul syzygies of $\mathfrak{m}$ : in other words, they generate the syzygies of the $R$-module $\mathfrak{m} \oplus \mathfrak{m} \oplus \mathfrak{m}$.

On the other hand, one has the obvious free resolution

$$
0 \rightarrow R(-3)^{3} \stackrel{\Psi}{\longrightarrow} R(-2)^{9} \longrightarrow R(-1)^{9} \longrightarrow \mathfrak{m} \oplus \mathfrak{m} \oplus \mathfrak{m} \rightarrow 0 .
$$

Splicing, we get the free complex

$$
0 \rightarrow R(-(d+3))^{3} \stackrel{\Psi(-d)}{\longrightarrow} R(-(d+2))^{9+d-2} \stackrel{\Phi}{\longrightarrow} R(-(d+1))^{d+5} \longrightarrow\left(J_{d+1}\right) \rightarrow 0 .
$$

Now use the Buchsbaum-Eisenbud acyclicity criterion: the ranks are easily seen to add up correctly. As for the heights of the required Fitting, one has ht $I_{3}(\Psi(-d))=$ ht $I_{3}(\Psi)=3$, as is obvious from (7), while one notes the shape of $\Phi$ as the $(d+5) \times(d+7)$ matrix

$$
\Phi=\left(\begin{array}{ccc|c}
K(\mathfrak{m}) & & & \widetilde{\mathcal{L}} \\
& K(\mathfrak{m}) & & \\
\hline & & K(\mathfrak{m}) & \mathcal{L}
\end{array}\right)
$$


where, to the left of the vertical line, $K(\mathfrak{m})$ stands for the first Koszul matrix of $\mathfrak{m}$ and the empty slots are all zeros. However, the matrix to the right of the vertical line is the $(d-2) \times(d+5)$ matrix of linear syzygies, as obtained above from the original syzygies of $J$, the lower matrix $\mathcal{L}$ coincides with the one described in (a) and the upper $\widetilde{\mathcal{L}}$ has certain linear coordinates.

Note that the diagonal sum of $K(\mathfrak{m})$ has only rank six, so it can only contribute this much to get a non-zero minor of size $(d+4) \times(d+4)$; forcefully, one has the use a full maximal minor of the right side matrix. It is clear that this patching allows for the construction of at least two $(d+4)$-minors with no proper common factor. This shows that ht $I_{d+4}(\Phi) \geqslant 2$, as required for the acyclicity criterion.

Remark 2.7. The result of item (b) also follows from [10, Theorem 2.1(ii)]. To see this, one observes that, in the notation there, $\sigma=d+1$, as follows from Lemma 2.3. Moreover, the condition on how a straight line intersects the fat scheme translates here, via Bézout theorem, into the inequality $\mu_{1}+\mu_{2} \leqslant d$, a condition that is always satisfied by a proper homaloidal type, as is the present case.

By that result, the image of the birational map is a smooth surface and, moreover, the surface is arithmetically Cohen-Macaulay [10, Proposition 2.4] (and hence also projectively normal). Since the present situation is more special it would be of some interest to have a direct algebraic proof of the Cohen-Macaulayness assertion. Unfortunately, the latter is at the moment outside the present scope.

\subsection{Further homological details}

As a sequel to the results of Theorem 2.6, we have the following structurally finer points.

Proposition 2.8. Keep the notation of Theorem 2.6 and, as introduced in the proof of the supplementary assertion of item (a), let $\mathfrak{L}$ denote the $(d-4+s) \times(d-2+s)$ submatrix of $\varphi$ omitting the first three rows. Then:

(i) the $R$-module $J / I$ is linearly presented by $\mathfrak{L}$ if $s=0$ (respectively, by its submatrix omitting the zero column if $s=1$ );

(ii) $J=I^{\text {sat }} \Rightarrow \operatorname{dim}(J / I)=0 \Rightarrow \mu_{i} \leqslant 2$, for all $i \Rightarrow(d ; \boldsymbol{\mu})=\left(4 ; 2^{3}, 1^{3}\right)$ or $(d ; \boldsymbol{\mu})=\left(5 ; 2^{6}\right) \Rightarrow$ $J=I^{\text {sat }}$, and hence all these conditions are mutually equivalent;

(iii) if $\operatorname{dim}(J / I)=1$, then $R / I$ is Cohen-Macaulay if and only if $J / I$ is Cohen-Macaulay; and

(iv) setting $\mathfrak{K}:=\operatorname{ker}(\mathfrak{L})$, the minimal syzygy matrix $\psi$ of $I$ is the submatrix of $\varphi \cdot \mathfrak{K}$ consisting of its first three rows.

Proof. (i) By the part of Theorem 2.6(a) which is independent of the assertion about the de Jonquières maps, we know that $s=1$ means that exactly one column of $\varphi$ is such that the entries on its first three rows are linear and the rest are zeros. Since the generators of $I$ are minimal generators of $J$, the result is clear.

(ii) Clearly, $\operatorname{dim}\left(I^{\text {sat }} / I\right)=0$, so the first implication of the stated string is obvious.

To proceed, by (i), one has ht $(I: J)=$ ht $I_{d-4+s}(\mathfrak{L})$ since $J / I$ and its zeroth Fitting ideal have the same radical. Therefore, $\operatorname{dim}(J / I)=\operatorname{dim}(R / I: J)=3-\mathrm{ht} I_{d-4+s}(\mathfrak{L})$, so we are to show that ht $I_{d-4+s}(\mathfrak{L})=3 \Rightarrow \mu_{i} \leqslant 2, \forall i$. Assuming the contrary, one has $\mu_{1} \geqslant 3$. Then $d-1+s-\mu_{1} \leqslant d-1+s-3=d-4+s$, and hence there is another reason for ht $I_{d-1+s-\mu_{1}}(\mathfrak{L}) \geqslant 3$. But letting $P_{1}$ denote the prime ideal for the first multiplicity, we see that $I_{d-1+s-\mu_{1}}(\mathfrak{L}) \subset P_{1}$ since otherwise we could invert a minor in the localization $R_{P_{1}}$ and thus have $J_{P_{1}}=P_{1}^{\mu_{1}}$ generated by $d-1+s-\left(d-1+s-\mu_{1}\right)=\mu_{1}$ elements - this is absurd as $P_{1}^{\mu_{1}}$ is minimally generated by $\mu_{1}+1$ elements.

We move on to the next implication, namely, that $\mu_{i} \leqslant 2$, for all $i$ can only happen for those two particular homaloidal types. This is an easy calculation using the equations of condition. 
Thus, letting $\left(d ; 2^{r_{2}}, 1^{r_{1}}\right)$ be the assumed type,

$$
\left\{\begin{array}{l}
r_{1}+2 r_{2}=3 d-3, \\
r_{1}+4 r_{2}=d^{2}-1 .
\end{array}\right.
$$

Manipulation of these equations yields $r_{1}=d(6-d)-5$, from which (since $r_{1} \geqslant 0$ ) the only possibilities are $d=4, r_{1}=3$ or, $d=5, r_{1}=0$; correspondingly, $r_{2}=3$ or $r_{2}=6$.

The last implication is well known: for $d=4$ the base ideal $I$ is always saturated (cf. [14, Theorem 1.5(i)]), while $\left(5 ; 2^{6}\right)$ is a classic (see, for example, [14, Theorem 2.14(iii) Supplement] and the preliminaries of the proof, where it is argued that $(x, y, z) f \in I$, where $f$ is the unique minimal generator of degree six of $J$ ).

(iii) This follows easily by chasing depth along the following structural exact sequences

$$
0 \rightarrow I \longrightarrow R \longrightarrow R / I \rightarrow 0 \text { and } 0 \rightarrow I \longrightarrow J \longrightarrow J / I \rightarrow 0
$$

using that $\operatorname{dim}(J / I)=1$, by hypothesis, and that $\operatorname{depth}(J)=2$ and $\operatorname{depth}(R)=3$.

(iv) Note that any syzygy of $\left\{D_{1}, D_{2}, D_{3}\right\}$ stacked on top of the $(d-4+s) \times 1$ zero matrix is a syzygy of $J$. Since $I=\left(D_{1}, D_{2}, D_{3}\right)$,

$$
\left(\frac{\psi}{\underline{\mathbf{0}}}\right)=\varphi \cdot \mathfrak{C}
$$

for a suitable matrix $\mathfrak{C}$ ('content' matrix), where the matrix on the left-hand side is the matrix $\psi$ of syzygies of $I$ stacked on top of a zero matrix with $(d-4+s)$ rows and same number of columns as $\psi$. Note that, in the case where $s=1$, this content matrix will involve the unit column $(10 \ldots 0)^{t}$. Clearly, $\operatorname{Im}(\mathfrak{C}) \subset \mathfrak{K}:=\operatorname{ker}(\mathfrak{L})$ : in other words, every column of $\mathfrak{C}$ is a syzygy of $\mathfrak{L}$.

We claim the reverse inclusion as well. To see this, let $\rho$ denote the submatrix of $\varphi$ with the first three rows. Then we wish to show that

$$
\left(D_{1} D_{2} D_{3}\right) \cdot \rho \cdot \mathfrak{K}=0 .
$$

Recall that $D_{j}$ is the minor of $\varphi$ omitting row $j$, for $j=1,2,3$. Thus, if $\tilde{\varphi}$ denotes the submatrix of $\varphi$ omitting its last row and $\mathfrak{Q}$ denotes the matrix of cofactors of $\tilde{\varphi}$, one has $\left(D_{1} D_{2} D_{3}\right)=L \cdot \mathfrak{Q}$, where $L$ denotes the last row of $\mathfrak{L}$.

Then

$$
\left(D_{1} D_{2} D_{3}\right) \cdot \rho \cdot \mathfrak{K}=L \cdot \mathfrak{Q} \cdot \rho \cdot \mathfrak{K}=\operatorname{det}(\tilde{\varphi}) \cdot L \cdot \mathfrak{K}=0,
$$

as required.

\section{Cremona maps with large highest multiplicity}

Some classical inequalities for Cremona map stem from the consideration of the three highest virtual multiplicities of the corresponding homaloidal type. As far as we know, not much however has been obtained by stressing the role of the behavior of the map in the neighborhood of a single point with highest virtual multiplicity.

The goal of this part is to point out substantial algebraic information by focusing on when the highest virtual multiplicity $\mu$ roughly lies on the interval $d-1 \geqslant \mu \geqslant\lceil d / 2\rceil$, with a big question mark about the subinterval $d-5 \geqslant \mu \geqslant\lceil d / 2\rceil$.

Applying an arithmetic quadratic transformation (see [1, Definition 5.2.3]) to a homaloidal type belonging to this imprecise family will often yield a homaloidal type of the same shape (informally, a 'telescopical' type), while in a few other instances it will afford a homaloidal type belonging to the inverse of the former. Thereby, use of the Hudson test repeatedly (or inductively) will show that these homaloidal types are proper. 


\subsection{Classes with saturated base ideal}

The first two families below are known from the literature (see, for example, [3, Example 2.9]), but not so much is known about the argument showing that these are the only families with the chosen value of the highest virtual multiplicity, nor the question of the saturation of the base ideal.

3.1.1. Highest virtual multiplicity $d-1$. The case where the highest virtual multiplicity is also the highest possible, namely, $\mu=d-1$, is well known. With this value of $\mu$, the remaining virtual multiplicities are forcefully all one (as follows immediately from the equations of condition). Thus, the corresponding homaloidal type is $\left(d ; d-1,1^{2 d-2}\right)$. Applying an arithmetic quadratic transformation based on the three highest multiplicities immediately gives $\left(d-1 ; d-2,1^{2(d-1)-2}\right)$, and hence induction gives that the type is proper. A Cremona map with this homaloidal type is the classical de Jonquières map. The homological features of a de Jonquières map are known (see, for example, [14, Theorem 2.7] and [22, Corollary 2.6]).

3.1.2. Highest virtual multiplicity $d-2$. Assume $d \geqslant 4$ to avoid a de Jonquières map of degree three.

We prove the following proposition.

Proposition 3.1. Let $T$ denote a proper homaloidal type of degree $d \geqslant 4$ and highest virtual multiplicity $d-2$. Then $T=\left(d ; d-2,2^{d-2}, 1^{3}\right)$. Moreover, given a plane Cremona map $F$ of degree $d \geqslant 4$, with this homaloidal type and with proper base points, $R / I$ is Cohen-Macaulay, where $I$ is the base ideal of $F$.

Proof. We first argue that any proper type $T$ with highest virtual multiplicity $d-2$ is necessarily of the stated form. Applying an arithmetic quadratic transformation based on the highest virtual multiplicities gives the virtual multiplicity $d-(d-2)-\mu_{2}=2-\mu_{2}$. Since $T$ is proper, this must be non-negative: that is, $\mu_{2} \leqslant 2$. Thus, for a proper type, necessarily $\mu_{i} \leqslant 2$ for every $i \geqslant 2$. Therefore, we can set $T=\left(d ; d-2,2^{s}, 1^{r-s}\right)$.

It is straightforward to deduce the following relations from the equations of condition.

$$
\left\{\begin{array}{l}
r+s=2 d-1, \\
r+3 s=4 d-5 .
\end{array}\right.
$$

The solution is straightforward: $s=d-2, r=d+1$.

To show that such a $T$ is proper, we apply an arithmetic quadratic transformation based on the virtual multiplicities $d-2,2,2$ (recall that $d \geqslant 4$ ). The result is the type $(d-2$; $d-4,2^{d-4}, 1^{3}$ ), and hence the Hudson test works telescopically, yielding the main inductive step. Therefore, it suffices to show that $\left(4 ; 2 ; 2^{4-2}, 1^{3}\right)=\left(4 ; 2^{3}, 1^{3}\right)$ is a proper type to start the induction. But this is obvious as an additional arithmetic quadratic transformation yields the homaloidal type $\left(2 ; 1^{3}\right)$ of a quadratic map.

Finally, we prove the last statement. To do so, let $p=p_{1}$ denote the point with virtual multiplicity $d-2$.

Since $F$ is not de Jonquières, by Proposition 2.4, the free resolution of the associated ideal $J$ of points with the virtual multiplicities of $F$ has the first of the two possible forms in Theorem 2.6: that is,

$$
0 \rightarrow R(-(d+2))^{d-2} \stackrel{\varphi}{\rightarrow} R(-(d+1))^{d-4} \oplus R(-d)^{3} \rightarrow J \rightarrow 0
$$

where $I=\left(J_{d}\right)$ is the base ideal of $F$.

We claim that $I_{1}(\varphi) \subset P$, where $P \subset R$ is the prime ideal of $p$. Indeed, otherwise, locally at $P$ one could invert an entry of $\varphi$ so that, by (9), the localization $J_{P}$ would be generated by 
at most $d-2$ elements. But this contradicts the fact that $J_{P}=P_{P}^{d-2}$ is minimally generated by $d-1$ elements because $P$ is generated by two linear forms (as we are assuming that $k$ is algebraically closed).

This implies, in particular, that $I_{1}(\mathfrak{L}) \subset P$, where $\mathfrak{L}$ denotes the $(d-4) \times(d-2)$ linear submatrix of $\varphi$, as in Proposition 2.8. Now we may assume at the outset that $P=(x, y)$. Since the entries of $\mathfrak{L}$ are linear forms, every such entry is actually a linear form in the ring $k[x, y]$. Viewed as a module over $k[x, y]$ the kernel of $\mathfrak{L}$ is a free module, necessarily of rank two. By flatness, this is also the nature of the kernel considered as a module over $R=k[x, y, z]$.

By Proposition 2.8, the minimal number of generators of the syzygy module of the base ideal $I$ of $F$ coincides with the minimal number of generators of the syzygy module of $\mathfrak{L}$. This shows that the minimal number of generators of the syzygy module of $I$ is two, and thus proves the statement.

\subsection{Classes with conjectured saturated base ideal}

3.2.1. Highest virtual multiplicity $d-3$. As in the previous case, we will face a strong restriction on the remaining virtual multiplicities. However, differently from the previous case where one has a unique class of proper homaloidal types, here an enriched family will pop up, subsuming subclasses distinguished according to the values of $d$ modulo three. The typical proper homaloidal type that will emerge has $3,2,1$ as remaining virtual multiplicities, with $d-3 \geqslant 3$.

Proposition 3.2. Let $T$ denote a proper homaloidal type of degree $d \geqslant 6$ and highest virtual multiplicity $d-3$. Then $T$ is one of the following, where $t \geqslant 2$.

(0) $d \equiv 0(\bmod 3)$.

Proper homaloidal types $(d=3 t)$ :

(0.1) $\left(d ; d-3,3^{2 t-2}, 2,1^{4}\right)$;

(0.2) $\left(d ; d-3,3^{2 t-3}, 2^{4}, 1\right)$.

(1) $d \equiv 1(\bmod 3)$.

Proper homaloidal types $(d=3 t+1)$ :

(1.1) $\left(d ; d-3,3^{2 t-1}, 2^{0}, 1^{5}\right)$;

(1.2) $\left(d ; d-3,3^{2 t-2}, 2^{3}, 1^{2}\right)$.

(2) $d \equiv 2(\bmod 3)$.

Proper homaloidal types $(d=3 t+2)$ :

(2.1) $\left(d ; d-3,3^{2 t-1}, 2^{2}, 1^{3}\right)$;

(2.2) $\left(d ; d-3,3^{2 t-2}, 2^{5}, 1^{0}\right)$.

(Conjectured supplement) Moreover, given a plane Cremona map $F$ of degree $d \geqslant 5$, with any of the stated homaloidal types and with proper base points, $R / I$ is Cohen-Macaulay, where $I$ is the base ideal of $F$.

Proof. We first argue that any proper type $T$ with highest virtual multiplicity $d-3$ is necessarily of the form $T=\left(d ; d-3,3^{r_{3}}, 2^{r_{2}}, 1^{r_{1}}\right)$. Since $T$ is proper and $\mu_{2} \leqslant d-3$, applying an arithmetic quadratic transformation based on the highest virtual multiplicities yields the virtual multiplicity $d-(d-3)-\mu_{2}=3-\mu_{2}$, which is non-negative only if $\mu_{2} \geqslant 3$. Thus, for a proper type, necessarily $\mu_{i} \leqslant 3$ for every $i \geqslant 2$. Therefore, we can set $T=\left(d ; d-3,3^{r_{3}}, 2^{r_{2}}, 1^{r_{1}}\right)$.

The equations of condition yield

$$
\left\{\begin{array}{l}
r_{1}+2 r_{2}+3 r_{3}+d-3=3 d-3 \\
r_{1}+4 r_{2}+9 r_{3}+(d-3)^{2}=d^{2}-1
\end{array}\right.
$$


An easy manipulation of these two relations yields

$$
\left\{\begin{array}{l}
r_{1}+r_{2}=5 \\
r_{2}+3 r_{3}=2 d-5 .
\end{array}\right.
$$

The solutions are straightforward by taking a non-negative integer partition of five and plugging into the second of these relations, which produces a congruence modulo three, whose solutions are pretty immediate.

To see that all these homaloidal types are proper, we apply the Hudson algorithm in terms of arithmetic quadratic transformations based on the three highest multiplicities (cf. [1, Corollary 5.2.21]. As above, in the case of highest multiplicity $d-1$ or $d-2$, the result of applying such an arithmetic transformation is telescopic. Indeed, for $t \geqslant 2$ the arithmetic transformation is exactly the same in all cases, as the three highest multiplicities are $d-3,3,3$. Moreover, since the degree goes down by three every time an arithmetic quadratic transformation is applied, the degree of the resulting type does not leave its class modulo three. Explicitly, one obtains the following cases.

Case $(0.1) \cdot\left(d-3 ; d-6,3^{2(t-2)}, 2,1^{4}\right)$.

Case $(0.2) .\left(d-3 ; d-6,3^{2 t-5}, 2^{4}, 1\right)(t \geqslant 3)$.

Case (1.1). $\left(d-3 ; d-6,3^{2 t-3}, 2^{0}, 1^{5}\right)$.

Case (1.2). $\left(d-3 ; d-6,3^{2(t-2)}, 2^{3}, 1^{2}\right)$.

Case $(2.1) .\left(d-3 ; d-6,3^{2 t-3}, 2^{2}, 1^{3}\right)$.

Case $(2.2) .\left(d-3 ; d-6,3^{2(t-2)}, 2^{5}, 1^{0}\right)$.

To start the induction, one needs the bottom type in each case, along with one additional arithmetic quadratic transformations $\mathcal{Q}$.

Case $(0.1) .\left(6 ; 3=6-3,3^{2}, 2,1^{4}\right)=\left(6 ; 3^{3}, 2,1^{4}\right) \stackrel{\mathcal{O}}{\sim}\left(3 ; 2,1^{4}\right)$ : de Jonquières.

Case $(0.2)$. $\left(6 ; 3=6-3,3,2^{4}, 1\right)=\left(6 ; 3^{2}, 2^{4}, 1\right) \stackrel{\mathcal{Q}}{\sim}\left(4 ; 2^{3}, 1^{3}\right)$ : the non-de Jonquières.

Case (1.1). $\left(7 ; 4=7-3,3^{3}, 2^{0}, 1^{5}\right)=\left(7 ; 4,3^{3}, 1^{5}\right) \stackrel{\mathcal{Q}}{\rightarrow}\left(4 ; 3,1^{6}\right)$ : de Jonquières.

Case (1.2). $\left(7 ; 4=7-3,3^{2}, 2^{3}, 1^{2}\right)=\left(7 ; 4,3^{2}, 2^{3}, 1^{2}\right) \stackrel{\mathcal{Q}}{\sim}\left(4 ; 2^{3}, 1^{3}\right)$ : the non-de Jonquières.

Case $(2.1)$. $\left(8 ; 5,3^{3}, 2^{2}, 1^{3}\right) \stackrel{\mathcal{Q}}{\sim}\left(5 ; 3,2^{3}, 1^{3}\right)$ : the saturated non-de Jonquières.

Case $(2.2) .\left(8 ; 5,3^{2}, 2^{5}\right) \stackrel{\mathcal{O}}{\sim}\left(5 ; 2^{6}\right)$ : the non-saturated.

This shows the properness of all types.

REMARK 3.3. Note that the virtual multiplicity two is present in all cases above, with the exception of (1.1). Thus, in all these cases, one has $\mu_{1}+2=d-3+2=d-1$. By [3, Proposition 5.2], this means that when the highest multiplicity is $d-3$, except for case (1.1), the Cremona map belongs to the closure of the set of Cremona maps of degree $d+1$.

3.2.2. Highest virtual multiplicity $d-4$. We first note that any proper homaloidal type $T$ with highest virtual multiplicity $d-4$ is necessarily of the form

$$
T=\left(d ; d-4,4^{r_{4}}, 3^{r_{3}}, 2^{r_{2}}, 1^{r_{1}}\right) .
$$

Indeed, since $T$ is proper and $\mu_{2} \leqslant d-4$, applying an arithmetic quadratic transformation based on the highest virtual multiplicities yields the virtual multiplicity $d-(d-4)-\mu_{2}=4-\mu_{2}$, which is non-negative only if $\mu_{2} \geqslant 4$. Thus, for a proper type, necessarily $\mu_{i} \leqslant 4$ for every $i \geqslant 2$.

We will, accordingly, assume this form at the outset in the next proposition. In addition, the bulk of this case will have $d-4 \geqslant 4$, that is, $d \geqslant 8$, though we harmlessly include $d=7$ as a sort of degenerate case. 
Proposition 3.4. Let $T=\left(d ; d-4,4^{r_{4}}, 3^{r_{3}}, 2^{r_{2}}, 1^{r_{1}}\right)$ denote a proper homaloidal type of degree $d$ and highest virtual multiplicity $d-4 \geqslant 3$. Then we have the following.

(a) $r_{3}$ and $r_{4}$ cannot vanish simultaneously.

(b) $r_{1}$ and $r_{2}$ cannot vanish simultaneously.

(c) If $r_{4}=0$, then $T$ is one of the types given by

$$
\left(7 ; 3=7-4,3^{3}, 2^{3}\right)=\left(7 ; 3^{4}, 2^{3}\right), \quad\left(8 ; 4=8-4,3^{5}, 1^{2}\right), \quad\left(10 ; 6=10-4,3^{7}, 1^{0}\right),
$$

where the second is obtained from the last by applying an arithmetic transformation on the three highest multiplicities.

(d) If $r_{3}=0$, then $r_{4}=\lfloor(d-3) / 2\rfloor \geqslant 2$ and

$$
T= \begin{cases}\left(d ; d-4,4^{\lfloor(d-3) / 2\rfloor}, 2^{3}, 1^{3}\right) & \text { if } d \text { is even, } \\ \left(d ; d-4,4^{(d-3) / 2}, 1^{7}\right) & \text { if } d \text { is odd and } \not \equiv 3 \bmod 4 .\end{cases}
$$

(e) Suppose that neither $r_{3}$ nor $r_{4}$ vanishes.

(e.1) $r_{1}=0, r_{2} \neq 0$ : in this case, $T=\left(d ; d-4,4^{(d-7) / 2}, 3^{3}, 2^{3}\right)$, where $d \geqslant 9$ is odd.

(e.2) $r_{2}=0, r_{1} \neq 0$ : in this case, $T=\left(d ; d-4,4^{\left(d-\left(r_{3}+3\right)\right) / 2}, 3^{r_{3}}, 1^{7-r_{3}}\right)$, where $d$ is even and $r_{3}=1,3,5$.

(Conjectured supplement) Moreover, given a plane Cremona map $F$ of degree $d \geqslant 7$, with any of the stated homaloidal types and with proper base points, $R / I$ is Cohen-Macaulay, where $I$ is the base ideal of $F$.

Proof. Consider the equations of condition for this type given by

$$
\left\{\begin{array}{l}
r_{1}+2 r_{2}+3 r_{3}+4 r_{4}=3 d-3-(d-4)=2 d+1, \\
r_{1}+4 r_{2}+9 r_{3}+16 r_{4}=d^{2}-1-(d-4)^{2}=8 d-17 .
\end{array}\right.
$$

An easy manipulation of these relations allows us to express $r_{1}$ and $r_{2}$ in terms of $r_{3}$ and $r_{4}$ and $d$ : that is,

$$
\left\{\begin{array}{l}
r_{1}=3 r_{3}+8 r_{4}-4 d+19 \\
r_{2}=3\left(d-3-\left(r_{3}+2 r_{4}\right)\right) .
\end{array}\right.
$$

Since $r_{1}$ and $r_{2}$ are non-negative, we have the inequalities

$$
3 r_{3}+8 r_{4} \geqslant 4 d-19, \quad r_{3}+2 r_{4} \leqslant d-3 .
$$

(a) This one is clear: $r_{3}=r_{4}=0$ imply $r_{1}=19-4 d<0$ for $d \geqslant 5$.

(b) Plugging $r_{1}=r_{2}=0$ into the expressions above yields two linear equations in $r_{3}, r_{4}$ in terms of $d$ which are readily solved, giving $2 r_{4}=d-10, r_{3}=d-3-(d-10)=7$. Then $d$ must be even and $d \geqslant 12$ since $r_{4}>0$ for $r_{1}=r_{2}=0$, as will be proven in the next item.

Note the telescopic behavior: that is,

$$
\left(d ; d-4,4^{(d-10) / 2}, 3^{7}\right) \stackrel{\mathcal{O}}{\sim}\left(d-4 ; d-8,4^{((d-4)-10) / 2}, 3^{7}\right),
$$

while for $d=12$,

$$
\left(12 ; 8,4,3^{7}\right) \stackrel{\mathcal{Q}}{\sim}\left(9 ; 5,3^{6}, 1\right) \stackrel{\mathcal{Q}}{\sim}\left(7 ; 3^{5}, 1^{2}\right) \stackrel{\mathcal{Q}}{\sim}\left(5 ; 3^{2} .1^{6}\right) \stackrel{\mathcal{O}}{\sim}(3 ;-1, \ldots) .
$$

Therefore, this homaloidal type is not proper.

(c) If $r_{4}=0$, then $(4 d-19) / 3 \leqslant r_{3} \leqslant d-3$. Necessarily, $d-3 \geqslant(4 d-19) / 3$ which means that $d \leqslant 10$. Now, for $8 \leqslant d \leqslant 10$, we see that $r_{3}=d-3$. Substituting for $r_{3}=d-3, r_{4}=0$ in the formulas for $r_{1}$ and $r_{2}$ yields $r_{1}=10-d, r_{2}=0$. 
The case where $d=10$ reduces to the case where $d=8$ by applying an arithmetic quadratic transformation, as one readily sees. Therefore, it suffices to check the latter. Applying such a transformation again yields the case (0.1) of Proposition 3.2, with $d=6$.

Now, as for $d=7$, the interval for $r_{3}$ allows for two possibilities: $r_{3}=3$ or $r_{3}=4$. In the first case, one gets the homaloidal type $\left(7 ; 3=7-4,3^{3}, 2^{3}, 1^{0}\right)=\left(7 ; 3^{4}, 2^{3}\right)$. Applying two successive arithmetic quadratic transformations yields the type $\left(3 ; 2,1^{4}\right)$ - a de Jonquières type. As for the case $r_{3}=4$, we get the type $\left(7 ; 3=7-4,3^{4}, 2^{0}, 1^{3}\right)=\left(7 ; 3^{4}, 1^{3}\right)$, then $\left(5 ; 3^{2}, 1^{6}\right)$; the latter is not a proper type since for $d=5$ there are only three proper homaloidal types (de Jonquières, symmetric $\left(5 ; 2^{6}\right)$ and $\left(5 ; 3,2^{3}, 1^{3}\right)$ ).

Finally, the case $d=9$ reduces to the above non-proper type in degree seven by an arithmetic quadratic transformation, and hence is not proper either.

(d) With $r_{3}=0$, the inequalities (12) imply that

$$
\frac{4 d-19}{8} \leqslant r_{4} \leqslant \frac{d-3}{2} .
$$

But the positive difference between these two bounds is $19 / 8-3 / 2=7 / 8<1$. This yields the stated value of $r_{4}$ as the floor of half $d-3$.

On the other hand, the value of $r_{2}$ is now given by $r_{2}=3\left(d-3-2 r_{4}\right)$, from which it follows that $r_{2}=3$ or $r_{2}=0$ depending on whether $d$ is even or odd, respectively.

Finally, plugging each of these values into the expression $r_{1}=8 r_{4}-4 d+19=\lfloor(d-3) / 2\rfloor-$ $4 d+19$ readily gives the two respective values of $r_{1}$, as stated.

To prove that both types are proper, one notes that, since $d \geqslant 7$, applying an arithmetic quadratic transformation to each of them yields a homaloidal type of the same shape by substituting $d-4$ for $d$. Explicitly, since $r_{4} \geqslant 2, d \geqslant 8$ and, further, the three highest virtual multiplicities are $d-4,4,4$. Thus, we get

$$
\begin{aligned}
(2 d-(d-4) & \left.-4-4 ; d-(d-4)-4=0, d-(d-4)-4=0, d-4-4,4^{\lfloor(d-3) / 2\rfloor-2}, \ldots\right) \\
& =\left(d-4 ; d-8,4^{\lfloor(d-4)-3) / 2\rfloor}, \ldots\right) .
\end{aligned}
$$

By recursion, according to the Hudson test, the two homaloidal types will be proper if and only if the resulting types of this operation for $7 \leqslant d \leqslant 11$ are proper (this is because we jump four at a time). One has:

$\left(11 ; 7.4^{4}, 1^{7}\right) \stackrel{\mathcal{Q}}{\sim}\left(7 ; 4^{2}, 3,1^{7}\right) \stackrel{\mathcal{O}}{\sim}(3 ;-1, \ldots):$ non-proper;

$\left(10 ; 6,4^{3}, 2^{3}, 1^{3}\right) \stackrel{\mathcal{O}}{\sim}\left(6 ; 4,2^{4}, 1^{3}\right)$ : proper by Proposition 3.1;

$\left(9 ; 5,4^{3}, 1^{7}\right) \stackrel{\mathcal{Q}}{\sim}\left(5 ; 4,1^{8}\right):$ de Jonquières;

$\left(8 ; 4^{3}, 2^{3}, 1^{3}\right) \stackrel{\mathcal{Q}}{\sim}\left(4 ; 2^{3}, 1^{3}\right)$ : the saturated non-de Jonquières.

This shows the statement of this item.

(e.1) Let $r_{1}=0$. Then the first of the basic relations in (11) yields $3 r_{3}+6 r_{4}=4 d-19-2 r_{4}$; upon substitution in the second relation, one obtains $r_{2}=2\left(r_{4}+5\right)-d$. Since $r_{2} \neq 0$, $d<2\left(r_{4}+5\right)$. Returning to the first relation gives $3 r_{3}+8 r_{4}<8\left(r_{4}+5\right)-19=8 r_{4}+21$, from which we deduce that $r_{3}<7$. But, again, the first of the relations in (11) forces four to divide $19+3 r_{3}$. The only possibility in the interval $1 \leqslant r_{3} \leqslant 6$ is $r_{3}=3$. This, in turn, implies that $r_{4}=(d-7) / 2$ so, in particular, $d$ is odd. Then also $r_{2}=2((d-7) / 2+5)-d=3$.

In conclusion, $T=\left(d ; d-4,4^{(d-7) / 2}, 3^{3}, 2^{3}\right)$. To show that it is proper, apply an arithmetic quadratic transformation to the three highest multiplicities; assuming $d \geqslant 11$ yields the same sort. At the bottom, one gets $\left(11 ; 7 ; 4^{2}, 3^{3} .2^{3}\right)$ which, in turn, eventually transforms to $\left(5 ; 3,2^{3}, 1^{3}\right)$, a well-known proper homaloidal type.

(e.2) Let $r_{2}=0$. From the second relation $r_{3}+2 r_{4}=d-3$, upon substitution into the first relation yields $r-1=10+2 r_{4}-d$ and, since $r_{1} \neq 0, d<10+2 r_{4}$. Returning to the second relation gives once more $r_{3}<7$. Also $r_{4}=\left(d-\left(r_{3}+3\right)\right) / 2$, so $r_{3}$ and $d$ must have opposite parities. Furthermore, $r_{1}=7-r_{3}$. 
This gives the claimed form of $T$. Changing by an arithmetic quadratic transformation is again telescopic, for $d \geqslant r_{3}+7$. At the bottom, we find $T=\left(r_{3}+7 ; r_{3}+3,4^{2}, 3^{r_{3}}, 1^{7-r_{3}}\right)$. Letting $r_{3}$ run through one to six we easily test the obtained types for properness by applying arithmetic quadratic transformations, and thus find that they are proper for $r_{3}$ odd and improper for $r_{3}$ even.

Remark 3.5. Again by [3, Corollary 5.2], except for the two cases in item (d), all such Cremona maps lie on the closure of Cremona maps of degree $d+1$.

Of course, this is pretty general: a proper homaloidal type whose highest virtual multiplicity is, say, $\mu_{1}=d-\ell$ divides into two classes of topological nature. The ones such that there exists a multiplicity $\mu_{i}=\ell-1$ correspond to Cremona maps of degree $d$ lying on the closure of the set of Cremona maps of degree $d+1$, while the rest give the union of the two classes, where either $\mu_{2}=\ell$ and $\mu_{i} \leqslant \ell-2$ for every $i \geqslant 3$, or $\mu_{i} \leqslant \ell-2$ for every $i \geqslant 2$. Neither case corresponds to Cremona maps of degree $d$ lying on the closure of the set of Cremona maps of degree $d+1$.

We close this part with a result showing that the line of argument used in the cases $\mu_{1} \leqslant d-2$ in order to prove saturation of the base ideal cannot be extended to the situation where $\mu_{1} \geqslant d-3$. First, we isolate the following more technical result, using the notation introduced in the proof of Propositions 2.8 and 3.1.

Lemma 3.6. Consider a Cremona map with homaloidal type $\left(d ; \mu_{1} \geqslant \ldots \geqslant \mu_{r}\right)$ and proper base points. Letting $P_{i} \subset R$ denote the prime ideal of the proper base point $p_{i}$, one has $I_{d-1-\mu_{i}}(\mathfrak{L}) \subset P_{i}$, for every $\mu_{i} \geqslant 3$. In particular, the Fitting ideal $I_{t}(\mathfrak{L})=2$ has codimension two in the range $d-1-\mu_{i} \leqslant t \leqslant d-4$.

Proof. If not, let there exist a $\left(d-1-\mu_{i}\right)$-minor of $\mathfrak{L}$ not belonging to some $P_{i}$. This minor is invertible in $R_{P_{i}}$, and hence the ideal $J_{P_{i}} \subset R_{P_{i}}$ is generated by $(d-1)-\left(d-1-\mu_{i}\right)=\mu_{i}$ elements, where $J \subset R$ denotes the corresponding ideal of fat points. But $J_{P_{i}}=P_{i_{P_{i}}}^{\mu_{i}}$ is generated by exactly $\mu_{i}+1$ elements, which yields a contradiction.

Now for the promised statement.

Proposition 3.7. Consider a Cremona map with homaloidal type $\left(d ; \mu_{1} \geqslant \ldots \geqslant \mu_{r}\right) \neq$ $\left(5 ; 2^{6}\right)$ and proper base points. The following condition are equivalent:

(a) $\mu_{1} \geqslant d-2$; and

(b) $I_{1}(\mathfrak{L})$ has codimension two.

Proof. The implication (a) $\Rightarrow$ (b) has already been explained in the proof of Proposition 3.1.

For the reverse implication, we may assume that $d \geqslant 5$ as, otherwise, (a) would be automatically satisfied. Note that $I_{1}(\mathfrak{L})$ is a prime ideal since it is generated by linear forms, and $I \subset I_{1}(\mathfrak{L})$. Therefore, it is a minimal prime of $R / I$, say, $I_{1}(\mathfrak{L})=P_{i}$ for some $1 \leqslant i \leqslant r$. In particular, $I \subset I_{d-4}(\mathfrak{L}) \subset P_{i}^{d-4}$, which implies that any form in $I_{d}$ goes through $p_{i}$ with multiplicity $\geqslant d-4$. Since $I_{d}$ defines a Cremona map, the general form in $I_{d}$ has multiplicity exactly $\mu_{i}$ on $p_{i}$. Therefore, $d-4 \leqslant \mu_{i}$, and hence also $d-4 \leqslant \mu_{1}$.

Let us contradict the assumption that $\mu_{1} \leqslant d-3$.

Recall that one is assuming that the type is not $\left(5 ; 2^{6}\right)$. Then, by the classification of types in Propositions 3.2 and 3.4 , if $d-4 \leqslant \mu_{1} \leqslant d-3$, then at least two different virtual multiplicities are greater than or equal to three. If it happens that one of these is $\mu_{i}$, we choose the other one, say, $\mu_{j}(j \neq i)$. Letting $P_{j}$ denote the corresponding prime ideal, we have two distinct base primes $P_{i}$ and $P_{j}$. 
By Lemma 3.6, the Fitting ideal $I_{d-1-\mu_{j}}(\mathfrak{L})$ has codimension two and $P_{j}$ is, necessarily, a minimal prime thereof.

On the other hand, we contend that $I_{d-1-\mu_{j}}(\mathfrak{L})$ is $P_{i}$-primary, which gives the contradiction that we seek. Actually, this is true of any $I_{t}(\mathfrak{L})$ such that $1 \leqslant t \leqslant d-4$.

Indeed, first note that $I_{t}(\mathfrak{L})$ has codimension two since it contains $I$. Now, say, $P_{i}=\left(\ell_{1}, \ell_{2}\right)$, two independent linear forms. Since the entries of $\mathfrak{L}$ are linear forms, the required operations to achieve these linear forms are all $k$-linear operations. This implies that a minor of arbitrary size is a polynomial on $\ell_{1}, \ell_{2}$ with coefficients in $k$. Thus, $P_{i}$ is the extension to $R$ of the maximal ideal of the subring $k\left[\ell_{1}, \ell_{2}\right] \subset R$ and $I_{t}(\mathfrak{L})$ is the extension to $R$ of an $\left(\ell_{1}, \ell_{2}\right)$-primary ideal of $k\left[\ell_{1}, \ell_{2}\right]$. Therefore, as an ideal of $R, I_{t}(\mathfrak{L})$ is a $P_{i}$-primary ideal: that is, $P_{i}$ is its unique associated prime.

\section{Cremona maps with small highest multiplicity}

\subsection{Preamble on non-saturated base ideals}

In the previous section we saw some evidence to the effect that non-saturated Cremona maps of degree $d$ and highest virtual multiplicity $>d-5$ do not exist, with the single exception of $d=5$ and highest virtual multiplicity $2=d-3$. Since being non-saturated means that the map has a non-perfect base ideal, it seems of some interest to find out if this question has any impact on the geometric side of the picture. In this part we essay a few steps in this direction.

We are actually compelled to pose the following question.

QUESTION 4.1. Let $\left(d ; \mu_{1}, \ldots\right)$ denote a proper homaloidal type and let $F$ stand for a Cremona map with this type and general base points. If the base ideal of $F$ is not saturated, then $\mu_{1} \leqslant\lfloor d / 2\rfloor$.

Note that, for a Cremona map, $\mu_{2} \leqslant\lfloor d / 2\rfloor$ always holds, so the relevant case is when $\mu_{1}>\mu_{2}$.

In other words, the question is whether the inequality $\mu_{1}>\lfloor d / 2\rfloor$ triggers saturation, which is a lot more ambitious than the conjectured result of the previous section. The gist of the present section rests on considering two conditions, apparently apart from each other. The first, as suggested, is to the effect that the highest virtual multiplicity be less than about half the degree of the map; the second requires that all virtual multiplicities be even numbers.

The next subsection deals with the second of these conditions, setting it up on a larger environment.

\subsection{The role of the symbolic square}

In this part we will focus on a set of virtual multiplicities $\boldsymbol{\mu}$ satisfying certain relations which are naturally related to the equations of condition of a Cremona map. Doubling these multiplicities will, in fact, satisfy the equations of condition. This is a purely arithmetic procedure, but it has some impact on the corresponding ideals of fat points.

Definition 4.2. Let $\boldsymbol{\mu}=\left\{\mu_{1}, \ldots, \mu_{r}\right\}$ be a set of non-negative integers satisfying the following condition: there exists an integer $s \geqslant 2$ such that

$$
\sum_{i=1}^{r} \mu_{i}=3(s-1) \quad \text { and } \quad \sum_{i=1}^{r} \mu_{i}^{2}=s(s-1) .
$$

Note that the first of the above relations implies that $s$ is uniquely determined. We will say that $\boldsymbol{\mu}$ is a sub-homaloidal multiplicity set in degree $s$. 
The reason for this terminology will soon become clear.

EXAmPLE 4.3. Let $\mu_{i}=\mu$ for all $i$, the so-called uniform case. Then the only solutions of the above equations are

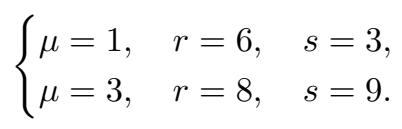

The calculation is straightforward: the two relations $r \mu=3(s-1), r \mu^{2}=s(s-1)$ readily imply $s=3 \mu$, which, in turn, yields the relation $r \mu=3(3 \mu-1)$. The latter is equivalent to $\mu(9-r)=3$, from which the two stated solutions follow.

EXAMPLE 4.4. Let $s \geqslant 3$ be an odd integer and let

$$
\mu_{1}=\ldots=\mu_{4}=(s-1) / 2, \quad \mu_{5}=\ldots=\mu_{s+3}=1
$$

(and thus, $r=s-1+4=s+3$ ).

The verification is immediate: $4(s-1) / 2+(s-1)=3(s-1)$, while $4(s-1)^{2} / 4+s-1=s^{2}-s$.

We will say that a set of non-negative integers $\boldsymbol{\nu}=\left\{\nu_{1}, \ldots, \nu_{r}\right\}$ is a homaloidal multiplicity set in degree $t$ if the corresponding type $(t ; \boldsymbol{\nu})$ is homaloidal: that is, satisfies the classical equations of condition for a Cremona map. The reason to emphasize this seemingly pedantic terminology is to be able to treat both multiplicity notions on the same footing and so, also, corresponding ideals with respect to the same set of points, in case the respective multiplicity sets have the same number.

Quite generally, the arithmetic as well as the geometric relation between the two are as follows.

Lemma 4.5. Let $\boldsymbol{\mu}=\left\{\mu_{1}, \ldots, \mu_{r}\right\}$ stand for a set of non-negative integers and let $s \geqslant 2$ denote an integer. The following conditions are equivalent:

(i) $\boldsymbol{\mu}$ is a sub-homaloidal multiplicity set in degree $s$; and

(ii) $2 \boldsymbol{\mu}$ is a homaloidal multiplicity set in degree $2 s-1$.

In addition, if we fix a set of points $\mathbf{p}$ with same cardinality as $\boldsymbol{\mu}$, then $I(\mathbf{p}, 2 \boldsymbol{\mu})=I(\mathbf{p}, \boldsymbol{\mu})^{(2)}$ (second symbolic power).

Proof. The proof of the equivalence is immediate, noting that $2(3 s-3)=3(2 s-1)-3$ and $4(s 2-s)=(2 s-1)^{2}-1$. The supplementary assertion follows since points in $\mathbb{P}^{n}$ are complete intersections and therefore the symbolic power of arbitrary order of the ideal of a point coincides with the respective ordinary power.

The following result shows a stronger tie between the two types above.

Proposition 4.6. Let $\boldsymbol{\mu}=\left\{\mu_{1} \geqslant \ldots \geqslant \mu_{r}\right\}$ denote a sub-homaloidal multiplicity set in degree $s \geqslant 3$ and let $\mathbf{p} \subset \mathbb{P}^{2}$ stand for a set of $r$ general points. Write $J:=I(\mathbf{p}, \boldsymbol{\mu})$. If the 'doubled' homaloidal type $(2 s-1 ; 2 \boldsymbol{\mu})$ is proper, then $\operatorname{indeg}(J)=s$.

Proof. Since the points in $\mathbf{p}$ are general, there is a Cremona map with homaloidal type $(2 s-1 ; 2 \boldsymbol{\mu})$ and these base points. Letting $\mathbb{J}:=I(\mathbf{p}, 2 \boldsymbol{\mu})$, by Lemma 2.3 , the initial degree of $\mathbb{J}$ is $2 s-1$, in the above notation.

First, it is clear that $\operatorname{indeg}(J) \geqslant s$, by using that $\mathbb{J}=J^{(2)}$ from Lemma 4.5. Indeed, if indeg $(J) \leqslant s-1$, then indeg $\left(J^{2}\right) \leqslant 2 s-2<2 s-1$ and hence also indeg(J) $<2 s-1$ as $J^{2} \subset J^{(2)}$. But this would contradict the result of Theorem 2.6, which says that indeg(J) $\geqslant 2 s-1$. 
For the reverse inequality, an easy calculation gives $e(R / J)=(1 / 2)(s+3)(s-1)$, and hence $\left(\begin{array}{c}s+2 \\ 2\end{array}\right)-e(R / J)=(s+5) / 2>0$. On the other hand, for any $t$,

$$
\operatorname{dim}_{k} J_{t} \geqslant \max \left\{\left(\begin{array}{c}
t+2 \\
2
\end{array}\right)-e(R / J), 0\right\} .
$$

Therefore, $\operatorname{dim}_{k} J_{s} \geqslant(s+5) / 2>0$. In particular, $\operatorname{indeg}(J) \leqslant s$, and hence equality must be the case, as stated.

The main focus of this section is the case where the homaloidal multiplicity set afforded by a sub-homaloidal multiplicity set is proper. The following examples show some erratic behavior of the second symbolic power of an ideal of fat points with a sub-homaloidal multiplicity set not affording a proper homaloidal multiplicity set. The calculations were carried out with the help of the Macaulay system, by taking points with random coordinates.

To avoid repetition, if $J$ is an ideal of fat points, then $\mathbb{J}$ will denote its symbolic square.

EXAmPLE 4.7. The multiplicity set $\left(4^{2}, 1^{10}\right)$ is sub-homaloidal in degree seven, as one easily checks. Here the doubled homaloidal type $\left(13 ; 8^{2}, 2^{10}\right)$ is not proper as $13<8+8$. Set $J=I\left(\mathbf{p} ; 4^{2}, 1^{10}\right)$ for general points $\mathbf{p}$. Then:

- $J$ is minimally generated in degrees seven and eight and the subideal $\left(J_{7}\right)$ has codimension one;

- $\mathbb{J}$ is generated in degrees thirteen, fourteen, fifteen and sixteen; and

- The ideal $\left(\mathbb{J}_{13}\right)$ has codimension one.

EXAMPLE 4.8. The multiplicity set $\left(4,2^{6}, 1^{2}\right)$ is sub-homaloidal in degree seven, as one easily checks. As it turns out, applying the Hudson test iteratively shows that the doubled homaloidal type $\left(13 ; 8,4^{6}, 2^{2}\right)$ is not proper. Setting $J$ and $\mathbb{J}$ as in the previous example, gives the following.

- $J$ is minimally generated in the single degree seven.

- $\mathbb{J}$ is generated in degrees thirteen and fourteen and its free resolution of $\mathbb{J}$ has same Betti numbers and twists as (6); in particular, $\mathbb{J}_{13}$ is a net.

- However, the ideal $\left(\mathbb{J}_{13}\right)$ still has codimension one.

- Canceling the gcd of the forms in $\mathbb{J}_{13}$ (the fixed part of the net) results in a nonhomaloidal net generating a saturated ideal.

EXAmple 4.9. The multiplicity set $\left(4,3^{6}, 1^{2}\right)$ is sub-homaloidal in degree nine and, once again, applying the Hudson test iteratively yields that the doubled homaloidal type $\left(17 ; 8,6^{6}, 2^{2}\right)$ is not proper. With the same notation, the following are obtained.

- $J$ is minimally generated in the single degree nine.

- $\mathbb{J}$ is generated in degrees seventeen and eighteen and its free resolution of $\mathbb{J}$ has same Betti numbers and twists as (6); in particular, $\mathbb{J}_{17}$ is a net.

- However, the ideal $\left(\mathbb{J}_{17}\right)$ still has codimension one.

- Canceling the gcd of the forms in $\mathbb{J}_{17}$ (the fixed part of the net) results in a nonhomaloidal net generating a non-saturated ideal.

- However, the latter non-saturated ideal fails for the template of Proposition 4.12 in that it admits minimal syzygies in two different degrees.

\subsection{Non-saturated base ideal: the underlying geometry}

An additional remarkable feature of a Cremona map $\mathfrak{F}: \mathbb{P}^{2} \rightarrow-\rightarrow \mathbb{P}^{2 *}$ is its characteristic matrix, which encapsulates the main geometric properties of the map. Using a pinhead description, the rows of the matrix, excluding the respective first entries, give the homaloidal type of the map and the types defining the principal curves corresponding to base points. This part of 
the paper will indirectly evoke features of the characteristic matrix, such as the notion of a principal curve, but, actually, no essential use will be made of its theory, except for a basic criterion to be found in [1] (our standing reference for an exhaustive account of this theory).

From now on, we will consider the situation of a sub-homaloidal multiplicity set in degree $s$ for which the highest multiplicity of the associated doubled homaloidal type is $s-1=\lfloor(2 s-1) / 2\rfloor$, as suggested at the beginning of the section.

We will use the terminology and notation of the previous subsection. Thus, $s \geqslant 3$ denotes an odd number.

Lemma 4.10. Let $\left(((s-1) / 2)^{3}, \mu_{4}, \ldots, \mu_{r}\right)$ denote a sub-homaloidal multiplicity set in degree $s \geqslant 3$. If the associated 'doubled' homaloidal set $\left((s-1)^{3}, 2 \mu_{4}, \ldots, 2 \mu_{r}\right)$ gives a proper homaloidal type in degree $2 s-1$ then $\left(s-1 ;((s-1) / 2)^{2},(s-3) / 2, \mu_{4}, \ldots, \mu_{r}\right)$ is a proper exceptional type.

Proof. Let us denote

$$
T:=\left(2 s-1 ;(s-1)^{3}, 2 \mu_{4}, \ldots, 2 \mu_{r}\right)
$$

and

$$
S:=\left(s-1 ;((s-1) / 2)^{2},(s-3) / 2, \mu_{4}, \ldots, \mu_{r}\right),
$$

where one may harmlessly assume that $\mu_{4} \geqslant \ldots \geqslant \mu_{r}>0$.

We first show that $S$ is an exceptional type. Namely, since $T$ is homaloidal,

$$
\left\{\begin{array}{l}
3(s-1)+2 \sum_{i \geqslant 4} \mu_{i}=6(s-1), \\
3(s-1)^{2}+4 \sum_{i \geqslant 4} \mu_{i}^{2}=4 s(s-1),
\end{array}\right.
$$

from which we derive $\sum_{i \geqslant 4} \mu_{i}=3(s-1) / 2$ and $\sum_{i \geqslant 4} \mu_{i}^{2}=\left(s^{2}+2 s-3\right) / 4$.

It follows that

$$
\left\{\begin{array}{l}
2(s-1) / 2+(s-3) / 2+\sum_{i \geqslant 4} \mu_{i}=3(s-1)-1, \\
2((s-1) / 2)^{2}+((s-3) / 2)^{2}+\sum_{i \geqslant 4} \mu_{i}^{2}=(s-1)^{2}-1,
\end{array}\right.
$$

as required.

Next, we look at properness. For $i \geqslant 0$, define $T_{i}$ and $S_{i}$, iteratively, by $T_{0}=T, S_{0}=S$ and for $i \geqslant 1, T_{i}$ (respectively, $S_{i}$ ) is the type obtained from $T_{i-1}$ (respectively, from $S_{i-1}$ ) by applying an arithmetic quadratic transformation based on three major multiplicities.

Since we are assuming that $T$ is a proper homaloidal type, $T_{j}=(1 ; \underbrace{0, \ldots, 0}_{r})$ for a certain $j \geqslant 1$. Let $T_{i}$ be any transformed type, with $i<j$.

Claim. $T_{i}$ is of the form $\left(2 t ; 2 \eta_{1}, \ldots, 2 \eta_{\sigma}, 1^{3}, 0^{r-\sigma-3}\right)$, for suitable $t \geqslant 1, \eta_{1} \geqslant \ldots \geqslant \eta_{\sigma} \geqslant 1$ and some $0 \leqslant \sigma \leqslant r-3$.

One proceeds by induction on $i \geqslant 1$. By applying an arithmetic quadratic transformation based on the first three multiplicities, it is easily seen that $T_{1}=\left(s+1 ; 2 \mu_{4}, \ldots, 2 \mu_{r}, 1^{3}\right)$. Note that now the degree is even and that the three highest multiplicities have been shifted to the end; after that, in every given stage, at least the smallest of the three highest multiplicities will be shifted to give place to a higher even multiplicity. 
Assume, inductively, that $T_{i-1}$ has the form $\left(2 t ; 2 \eta_{1}, \ldots, 2 \eta_{\sigma}, 1^{3}, 0^{r-\sigma-3}\right)$ and let us show that, necessarily, $\sigma \geqslant 3$. Indeed, first is obvious that $\sigma \neq 0$ as, otherwise, $T_{i-1}=\left(2 ; 1^{3}\right)$, and hence $T_{i}=(1 ; 0, \ldots, 0)$, which would contradict our assumption. If $\sigma=1$, then the first equation of condition gives $2 \eta_{1}+3=3.2 t-3=6 t-3$. In addition, one knows that the sum of the highest multiplicities does not exceed the degree, and hence $2 \eta_{1}+1 \leqslant 2 t$. Together this yields $t \leqslant 1$, and thus implies that $\eta_{1}=0$. However, this contradicts the assumption that $\eta_{1}>0$.

The case where $\sigma=2$ is similar: $t \geqslant \eta_{1}+\eta_{2}=3 t-3$ still yields $t=1$, forcing $\eta+1+\eta_{2} \leqslant 1$ : that is, $\eta_{2}=0$, which is a contradiction.

Now, since $\sigma \geqslant 3$, one sees that the transformed type is

$$
\left(2\left(2 t-\eta_{1}-\eta_{2}-\eta_{3}\right) ; 2\left(t-\eta_{2}-\eta_{3}\right), 2\left(t-\eta_{1}-\eta_{3}\right), 2\left(t-\eta_{1}-\eta_{2}\right), 2 \eta_{4}, \ldots, 2 \eta_{\sigma}, 1^{3}, 0 \ldots, 0\right),
$$

where the first three multiplicities are in decreasing order, but need to be reordered as compared with the subsequent multiplicities $2 \eta_{4} \geqslant \ldots \geqslant 2 \eta_{\sigma}$. In fact, some of the first three written above may turn out to vanish, so increasing the number of zeros and decreasing $\sigma$. However, since for $i \geqslant 1$ both the degree and the multiplicity are even, the number of ones will not change. The number of zeros is a consequence of the fact that the total number of multiplicities does not change under an arithmetic quadratic transformation.

This proves the claim.

Next, according to [1, Proposition 5.5.5], an exceptional type is proper if and only it is ultimately transformable into a type $(0 ;-1, \underbrace{0, \ldots, 0}_{r-1})$ after a finite number of arithmetic quadratic transformations.

$$
\text { ClAIM. If } T_{i}=\left(2 t ; 2 \eta_{1}, \ldots, 2 \eta_{\sigma}, 1^{3}, 0^{r-\sigma-3}\right) \text {, then } S_{i}=\left(t ; \eta_{1}, \ldots, \eta_{\sigma}, 1^{2}, 0^{r-\sigma-2}\right) \text {. }
$$

The proof is again by induction on $i$ and is similar to the previous one, so we only mark the main points. Thus, one easily sees that $S_{1}=\left((s+1) / 2 ; \mu_{4}, \ldots, \mu_{r}, 1^{2}, 0\right)$. Supposing that $S_{i-1}=\left(t ; \eta_{1}, \ldots, \eta_{\sigma}, 1^{2}, 0^{r-\sigma-2}\right)$ the next stage will have the form

$$
\left(2 t-\eta_{1}-\eta_{2}-\eta_{3} ; t-\eta_{2}-\eta_{3}, t-\eta_{1}-\eta_{3}, t-\eta_{1}-\eta_{2}, \eta_{4}, \ldots, \eta_{\sigma}, 1^{2}, 0 \ldots, 0\right) .
$$

This time there may appear more ones, but the number of zeros is always the number of zeros in $T_{i+1}$ plus one. So much for the claim.

In particular, since in the one before the last stage of transforming $T$ one gets the type $\left(2 ; 1^{3}, 0^{r-3}\right)$, the corresponding transformed type of $S$ is $\left(1 ; 1^{2}, 0^{r-2}\right)$. The latter clearly transforms to $\left(0 ;-1,0^{r-1}\right)$, as desired.

The main result of this part is as follows.

TheOrem 4.11. Let $\left(((s-1) / 2)^{3}, \mu_{4}, \ldots, \mu_{r}\right)$ denote a sub-homaloidal multiplicity set in degree $s \geqslant 3$. Assume that the associated 'doubled' homaloidal set $\left((s-1)^{3}, 2 \mu_{4}, \ldots, 2 \mu_{r}\right)$ gives a proper homaloidal type in degree $2 s-1$. Let $I \subset R=k[x, y, z]$ denote the base ideal of a Cremona map $\mathfrak{F}$ on $r$ general points having this homaloidal type. Then there are three principal curves of $\mathfrak{F}$ of degree $s-1$ and three independent linear forms $\ell_{1}, \ell_{2}, \ell_{3} \in R$ such that $I=\left(\ell_{3} f_{1} f_{2}, \ell_{2} f_{1} f_{3}, \ell_{1} f_{2} f_{3}\right)$.

Proof. Let $\left\{P_{1}, \ldots, P_{r}\right\} \subset R$ denote the defining prime ideals of $r$ general points over which $\mathfrak{F}$ is a Cremona map with homaloidal type $T:=\left(2 s-1 ;\left((s-1)^{3}, 2 \mu_{4}, \ldots, 2 \mu_{r}\right)\right.$. We may assume that the first three points are $(0: 0: 1),(0: 1: 0),(1: 0: 0)$. From Theorem 2.6 we know that the base ideal $I \subset R$ of $\mathfrak{F}$ is generated by the homogeneous piece of degree $2 s-1$ 
of the ideal of fat points

$$
\mathbb{J}:=J^{(2)}=P_{1}^{s-1} \cap P_{2}^{s-1} \cap P_{3}^{s-1} \cap P^{2 \mu_{4}} \cap \ldots \cap P^{2 \mu_{r}},
$$

where $J:=P_{1}^{(s-1) / 2} \cap P_{2}^{(s-1) / 2} \cap P_{3}^{(s-1) / 2} \cap P^{\mu_{4}} \cap \ldots \cap P^{\mu_{r}}$.

Consider the following additional ideals of fat points:

$$
\begin{aligned}
& J_{1}=P_{1}^{(s-3) / 2} \cap P_{2}^{(s-1) / 2} \cap P_{3}^{(s-1) / 2} \cap P_{4}^{\mu_{4}} \ldots \cap P_{r}^{\mu_{r}} ; \\
& J_{2}=P_{1}^{(s-1) / 2} \cap P_{2}^{(s-3) / 2} \cap P_{3}^{(s-1) / 2} \cap P_{4}^{\mu_{4}} \ldots \cap P_{r}^{\mu_{r}} \\
& J_{3}=P_{1}^{(s-1) / 2} \cap P_{2}^{(s-1) / 2} \cap P_{3}^{(s-3) / 2} \cap P_{4}^{\mu_{4}} \ldots \cap P_{r}^{\mu_{r}} .
\end{aligned}
$$

By Lemma 4.10, the respective types of $J_{1}, J_{2}, J_{3}$ are proper exceptional types in degree $s-1$. By [1, Proposition 5.5.13], for each $i=1,2,3$, there exists a non-zero irreducible homogeneous polynomial $f_{i} \in J_{i}$ of degree $s-1$ which defines a principal curve of $\mathfrak{F}$. Since we are assuming that $P_{1}=(x, y), P_{2}=(x, z), P_{3}=(y, z)$, the forms $x f_{1} f_{2}, z f_{1} f_{3}, y f_{2} f_{3}$ belong to $\mathbb{J}$. But they are of degree $2 s-1$, and hence $\left(x f_{1} f_{2}, z f_{1} f_{3}, y f_{2} f_{3}\right) \subset I$. On the other hand, no two $f_{i}, f_{j}(i \neq j)$ have a proper common factor as, otherwise, $f_{i} \in J$, while the latter has initial degree $s$, by Proposition 4.6. Therefore, for example, $\left\{f_{1}\left(x f_{2}+y f_{3}\right), z f_{2} f_{3}\right\}$ have no proper common factors, and hence the ideal $\left(x f_{1} f_{2}, z f_{1} f_{3}, y f_{2} f_{3}\right)$ has codimension two. By the same token, $\left\{x f_{1} f_{2}, z f_{1} f_{3}, y f_{2} f_{3}\right\}$ are $k$-linearly independent. The equality now follows.

Proposition 4.12. The notation is as in Theorem 4.11. Then

(a) $I$ is a non-saturated ideal with minimal graded resolution

$$
0 \rightarrow R(-3 s) \stackrel{\psi}{\longrightarrow} R^{3}(-(3 s-1)) \stackrel{\varphi}{\longrightarrow} R^{3}(-(2 s-1)) \rightarrow I \rightarrow 0
$$

where

$$
\varphi=\left(\begin{array}{ccc}
\ell_{2} f_{3} & -\ell_{3} f_{3} & 0 \\
-\ell_{1} f_{1} & 0 & \ell_{3} f_{1} \\
0 & \ell_{1} f_{2} & -\ell_{2} f_{2}
\end{array}\right) \quad \text { and } \quad \psi=\left(\begin{array}{l}
\ell_{3} \\
\ell_{2} \\
\ell_{1}
\end{array}\right)
$$

(b) $I^{\text {sat }}=\left(I, f_{1} f_{2} f_{3}\right)$.

Proof. (a) The maps are easily seen to give a complex. A simple application of the Buchsbaum-Eisenbud acyclicity criterion shows the contention.

(b) Since $I$ is the base ideal of a Cremona map, $I_{2 s-1}^{\text {sat }}=I_{2 s-1}$ and $I_{d}^{\text {sat }}=0$ for $d<2 s-1$. By part (a) and [14, Proposition 1.3(ii)], and as $R / I^{\text {sat }}$ is Cohen-Macaulay, it follows that $I^{\text {sat }}$ is minimally generated by four forms, three of which can be taken to be the minimal generators of $I$ as in (a) and the fourth is some form of degree $3(s-1)$.

Consider the matrix

$$
\left(\begin{array}{ccc}
f_{1} & 0 & 0 \\
0 & f_{2} & 0 \\
0 & 0 & f_{3} \\
\ell_{3} & \ell_{2} & \ell_{1}
\end{array}\right)
$$

Its three-minors form a minimal set of generators of the ideal $\left(I, f_{1} f_{2} f_{3}\right)$, since, for any $i \neq j$, one has $\operatorname{gcd}\left(f_{i}, f_{j}\right)=1$. This ideal is contained in $I^{\text {sat }}$ because $(x, y, z) f_{1} f_{2} f_{3}=$ $\left(\ell_{1}, \ell_{2}, \ell_{3}\right) f_{1} f_{2} f_{3} \in I$. Since $\operatorname{deg} f_{1} f_{2} f_{3}=3(s-1)$, the proof is complete. 


\section{References}

1. M. Alberich-Carramiñana, Geometry of the Plane Cremona Maps, Lecture Notes in Mathematics 1769 (Springer, Berlin-Heidelberg, 2002).

2. C. Bisi, A. Calabri and M. Mella, 'On plane Cremona transformations of fixed degree', J. Geom. Anal. 25 (2015) 1108-1131.

3. J. Blanc and A. Calabri, 'On degenerations of plane Cremona transformations', Math. Z. 282 (2016) 223-245.

4. C. Ciliberto, M. A. Cueto, M. Mella, K. Ranestad and P. Zwiernik, 'Cremona linearizations of some classical varieties', Proceedings of the Conference "Homage to Corrado Segre", Torino, Italy, 2014, Trends in the History of Science (Birkhäuser, Basel) to appear, arXiv:1403.1814v1 [math.AG].

5. C. Ciliberto, F. Russo and A. Simis, 'Homaloidal hypersurfaces and hypersurfaces with vanishing Hessian', Adv. Math. 218 (2008) 1759-1805.

6. B. Costa and A. Simis, 'New constructions of Cremona maps', Math. Res. Lett. 20 (2013) 629-645.

7. E. D. Davis, A. V. Geramita and P. Maroscia, 'Perfect homogeneous ideals: Dubreil's theorems revisited', Bull. Sci. Math., 2e série 108 (1984) 143-185.

8. A. Doria, H. Hassanzadeh and A. Simis, 'A characteristic free criterion of birationality', Adv. Math. 230 (2012) 390-413.

9. M. Dumnicki, T. Szemberg and H. Tutaj-Gasińska, 'A vanishing theorem and symbolic powers of planar point ideals', LMS J. Comput. Math. 16 (2013) 373-387.

10. A. V. Geramita, A. Gimigliano and Y. Pitteloud, 'Graded Betti numbers of some embedded rational $n$-folds', Math. Ann. 301 (1995) 363-380.

11. A. V. Geramita and F. Orecchia, 'Minimally generating ideals defining certain tangent cones', J. Algebra 78 (1982) 36-57.

12. E. Guardo and B. Harbourne, 'Resolutions of ideals of six fat points in $\mathbb{P}^{2}$, J. Algebra 318 (2007) 619-640.

13. B. Harbourne, 'Free resolutions of fat point ideals on $\mathbb{P}^{2}$ ', J. Pure Appl. Algebra 125 (1998) 213-234.

14. S. H. Hassanzadeh and A. Simis, 'Plane Cremona maps: saturation, regularity and fat ideals', J. Algebra 371 (2012) 620-652.

15. R. Miranda, 'Linear systems of plane curves', Notices Amer. Math. Soc. 46 (1999) 192-202.

16. M. Nagata, 'On rational surfaces II, Memoirs of the College of Sciences', Univ. Kyoto, Ser. A 33 (1960) $271-293$.

17. I. PAn, 'Sur le multidegré des transformations de Cremona', C. R. Acad. Sci. Paris 330 (2000) no. Série I, 297-300.

18. I. PAN, 'Les transformations de Cremona stellaires', Proc. Amer. Math. Soc. 129 (2001) 1257-1262.

19. I. PAN and F. Russo, 'Cremona transformations and special double structures', Manuscripta Math. 117 (2005) 491-510.

20. I. PAN and A. Simis, 'Cremona maps of de Jonquières type', Canad. J. Math. 67 (2015) 923-941.

21. A. Simis, 'Cremona transformations and some related algebras', J. Algebra 280 (2004) no. 1, 162-179.

22. A. Simis and S. Tohăneanu, 'The ubiquity of Sylvester forms in almost complete intersections', Collect. Math. 66 (2015) 1-31.

23. A. Simis and W. V. Vasconcelos, 'The syzygies of the conormal module', Amer. J. Math. 103 (1981) 203-224.

24. A. Simis and R. H. Villarreal, 'Linear syzygies and birational combinatorics', Results Math. 48 (2005) no. 3-4, 326-343.

25. A. Simis and R. H. Villarreal, 'Combinatorics of Cremona monomial maps', Math. Comp. 81 (2012) 1857-1867.

Zaqueu Ramos
Departamento de Matemática, CCET
Universidade Federal de Sergipe
49100-000 São Cristovão
Sergipe
Brazil
zaqueu.ramos@gmail.com

\author{
Aron Simis \\ Departamento de Matemática, CCEN \\ Universidade Federal de Pernambuco \\ 50740-560 Recife, PE \\ Brazil \\ and \\ Departamento de Matemática, CCEN \\ Universidade Federal da Paraíba \\ 58059-900 João Pessoa, PB \\ Brazil \\ aron@dmat.ufpe.br
}

